\title{
de Sitter quantum loops as the origin of primordial non-Gaussianities
}

\author{
Haipeng An, ${ }^{1}$ Mark B. Wise, ${ }^{2}$ and Zipei Zhang ${ }^{1}$ \\ ${ }^{1}$ Department of Physics, Tsinghua University, Beijing 100084, China \\ ${ }^{2}$ Walter Burke Institute for Theoretical Physics, California Institute of Technology, \\ Pasadena, California 91125, USA
}

(Received 18 September 2018; published 15 March 2019)

\begin{abstract}
It was recently pointed out that in some inflationary models quantum loops containing a scalar of mass $m$ that couples to the inflaton can be the dominant source of primordial non-Gaussianities. We explore this phenomenon in the simplest such model focusing on the behavior of the primordial curvature fluctuations for small $m / H$. Explicit calculations are done for the three- and four-point curvature fluctuation correlations. Constraints on the parameters of the model from the cosmic microwave background limits on primordial non-Gaussianity are discussed. The bispectrum in the squeezed limit and the trispectrum in the compressed limit are examined. The form of the $n$-point correlations as any partial sum of wave vectors gets small is determined.
\end{abstract}

DOI: $10.1103 /$ PhysRevD.99.056007

\section{INTRODUCTION}

An inflationary era in the early Universe is a popular possibility for the solution of the horizon and flatness problems [1]. It also provides an elegant mechanism to generate primordial density perturbations that, in the early Universe, have wavelengths well outside the horizon. In the most simple inflationary cosmology where only a single inflaton field with a standard kinetic term plays a role, the density perturbations are almost Gaussian and any nonGaussianities will be unobservable for the foreseeable future [2].

The inflationary era occurs in the early Universe when the energy density is (temporarily) dominated by vacuum energy, resulting in a scale factor that expands exponentially with time. This exponential expansion inflates the size of regions that were in causal contact to enormous size, solving the horizon problem. It also exponentially increases the physical wavelength of perturbations with fixed comoving wavelength, causing the density perturbations that arise from quantum fluctuations and are relevant for large-scale structure and the cosmic microwave background radiation (CMB) to have wavelengths that are well outside the horizon when inflation ends. At the end of the inflationary era the Universe reheats to a conventional radiation (or matter) dominated universe.

Published by the American Physical Society under the terms of the Creative Commons Attribution 4.0 International license. Further distribution of this work must maintain attribution to the author(s) and the published article's title, journal citation, and DOI. Funded by SCOAP .
Galaxies are biased objects, and as such the power spectrum for fluctuations ${ }^{1}$ in their number density can be enhanced, at low wave vectors, if there are non-Gaussian primordial curvature correlations (generated in the inflationary era) that are enhanced as a single wave vector or partial sum of wave vectors go to zero [3-6]. For the three- and four-point primordial curvature correlations the dominant enhancements occur in the squeezed and compressed limits, respectively.

These enhancements in the galaxy number density power spectrum (and bispectrum [7]) at very small wave vectors are sometimes called scale-dependent biases and have been studied extensively in quasi-single field inflation (QSFI [8]), which contains an additional scalar degree of freedom $s$ with mass $m$. They cannot arise from nonlinear gravitational evolution [9].

In QSFI the primordial non-Gaussianity results from tree diagrams in this theory. Recently, a model was constructed where it is quantum loop diagrams in de Sitter space that give rise to the non-Gaussian correlations that enhance the galaxy number density power spectrum at small wave vectors [10].

In this paper we follow up on this observation by considering the simplest model where loop diagrams dominate the non-Gaussianities. This model contains the inflaton field and an additional massive scalar $s$ with mass $m$ that we take to be small compared with the Hubble constant during inflation. A $Z_{2}$ symmetry in the system forbids the tree-level contribution from $s$ to the correlation functions of the curvature perturbation. We compute the

\footnotetext{
${ }^{1}$ A similar phenomena happens for the galaxy number density bispectrum.
} 
curvature three-point correlation (bispectrum) and fourpoint correlation (trispectrum) for general wave vectors. When all ratios of wave vectors are not unusually large or small, the bispectrum and trispectrum have the same form as local non-Gaussianity and $\tau_{N L}$ non-Gaussianity, respectively. ${ }^{2}$ In the squeezed and compressed limits the bispectrum and trispectrum have the familiar enhancements that give rise to an enhanced galaxy power spectrum at low wave vectors. We also discuss the form of the $n$-point curvature fluctuations as the partial sum of wave vectors goes to zero. We plot the enhancement of the two-point power spectrum of the galactic halo distributions generated by the quantum loops in this model using a simplified threshold model [12] for the galaxy halo number density.

The rest of the paper is organized as follows. In Sec. II we discuss the details of the model. In Sec. III we present the general three- and four-point correlation functions of the curvature perturbation. Details of the calculations are relegated to the appendices. In Sec. IV we calculate the constraints on the parameters of the model from CMB limits on the primordial bispectrum and trispectrum $[11,13]$. In contrast to most models, the constraint from the limit on the trispectrum is stronger. We conclude in Sec. V.

\section{THE MODEL}

The model contains two scalar fields: the inflaton $\phi$ and another scalar field $s$ of mass $m$. We assume that the inflation and $s$ fields have a $\phi \rightarrow-\phi$ symmetry and $s \rightarrow-s$ symmetry. We also assume a shift symmetry for $\phi$ that is only broken by the inflation potential. Then, up to the lowest-dimension term that contains interactions of $\phi$ with $s$ the Lagrangian can be written as

$$
\begin{aligned}
\mathcal{L}= & \frac{1}{2} g^{\mu \nu} \partial_{\mu} \phi \partial_{\nu} \phi\left(1+\frac{s^{2}}{2 \Lambda^{2}}\right)-V(\phi) \\
& +\frac{1}{2} g^{\mu \nu} \partial_{\mu} s \partial_{\nu} s-\frac{1}{2} \mu^{2} s^{2},
\end{aligned}
$$

where $V$ is the potential of $\phi$, which drives the slow-roll of $\phi$ during inflation. During inflation $\phi$ has a background value $\phi_{0}(t)$ that depends on time. Its magnitude relative to $H^{2}$ is fixed by the $\mathrm{CMB}$ temperature fluctuations to be $\dot{\phi}_{0} / H^{2} \simeq 3.5 \times 10^{4}$.

This model has a fine-tuning. The physical $s$ mass $m$ is a sum of two terms: one is the mass parameter $\mu$ from the $s$ potential, and the other is from the interaction above. Explicitly,

$$
m^{2}=\mu^{2}-\frac{\dot{\phi}_{0}^{2}}{4 \Lambda^{2}}
$$

\footnotetext{
${ }^{2} \mathrm{~A}$ trispectrum with $\tau_{N L}$ non-Gaussianity satisfies Eq. (21) of Ref. [11] with $g_{N L}=0$.
}

To get the enhancements we mentioned in the Introduction we need $m / H<1$, where $H$ is the Hubble constant during inflation. But to get observable non-Gaussianities we need the second term in the equation above to be much greater than $H^{2}$ (hence the tuning). Finally, we assume that there exists an inflaton potential that gives an acceptable region in $r-n_{s}$ space and is flat enough that $m$ can be approximated as a constant for the calculation of the non-Gaussianities generated during inflation.

We proceed along the lines of the effective field theory of inflation and work in the gauge where $\phi(x)=\phi_{0}(t)$ [14]. A Goldstone mode $\pi$, due to the breaking of the timetranslation symmetry, is introduced to describe the curvature perturbation as follows:

$g^{\mu \nu} \partial_{\mu} \phi \partial_{\nu} \phi \rightarrow g^{00} \dot{\phi}_{0}^{2} \rightarrow \dot{\phi}_{0}^{2} g^{\mu \nu} \partial_{\mu}\left(t+\pi / \dot{\phi}_{0}\right) \partial_{\nu}\left(t+\pi / \dot{\phi}_{0}\right)$,

where the evolution of $\dot{\phi}_{0}$ is neglected and the relation between $\pi$ and the curvature perturbation $\zeta$ in this model can be written as

$$
\zeta=-\frac{H}{\dot{\phi}_{0}} \pi
$$

A three-point and a four-point interaction between $\pi$ and $s$ are induced with

$\mathcal{L}_{\text {int }}=-\frac{1}{2 H^{3} \tau^{3}} \frac{\dot{\phi}_{0}}{\Lambda^{2}} \frac{\partial \pi}{\partial \tau} s^{2}+\frac{1}{4 H^{2} \tau^{2} \Lambda^{2}} \eta^{\mu \nu} \partial_{\mu} \pi \partial_{\nu} \pi s^{2}$,

where $\tau$ is the conformal time defined as $\tau=-e^{-H t} / H$ and $\eta^{\mu \nu}=\operatorname{diag}(1,-1,-1,-1)$. Here we include the $\sqrt{-g}$ factor in the Lagrangian.

The mode expansions of $\pi$ and $s$ are defined as

$$
\begin{array}{r}
\pi(\vec{x}, \tau)=\int \frac{d^{3} k}{(2 \pi)^{3}}\left[\pi_{k}(\tau) e^{i \vec{k} \cdot \vec{x}} a_{\vec{k}}+\pi_{k}^{*}(\tau) e^{-i \vec{k} \cdot \vec{x}} a_{\vec{k}}^{\dagger}\right], \\
s(\vec{x}, \tau)=\int \frac{d^{3} k}{(2 \pi)^{3}}\left[s_{k}(\tau) e^{i \vec{k} \cdot \vec{x}} b_{\vec{k}}+s_{k}^{*}(\tau) e^{-i \vec{k} \cdot \vec{x}} b_{\vec{k}}^{\dagger}\right], \\
{\left[a_{\vec{k}}, a_{\vec{k}^{\prime}}^{\dagger}\right]=\left[b_{\vec{k}}, b_{\vec{k}^{\prime}}^{\dagger}\right]=(2 \pi)^{3} \delta\left(\vec{k}-\vec{k}^{\prime}\right) .}
\end{array}
$$

The mode functions $\pi_{k}$ and $s_{k}$ satisfy the equations of motion

$$
\begin{array}{r}
\frac{\partial}{\partial \tau}\left(\frac{1}{H^{2} \tau^{2}} \frac{\partial \pi_{k}}{\partial \tau}\right)+\frac{k^{2} \pi_{k}}{H^{2} \tau^{2}}=0 \\
\frac{\partial}{\partial \tau}\left(\frac{1}{H^{2} \tau^{2}} \frac{\partial s_{k}}{\partial \tau}\right)+\frac{k^{2} s_{k}}{H^{2} \tau^{2}}+\frac{m^{2} s_{k}}{H^{4} \tau^{4}}=0
\end{array}
$$

and the Wronskian invariance 
$\frac{\pi_{k}}{H \tau} \frac{d}{d \tau} \frac{\pi_{k}^{*}}{H \tau}-\frac{\pi_{k}^{*}}{H \tau} \frac{d}{d \tau} \frac{\pi_{k}}{H \tau}=\frac{s_{k}}{H \tau} \frac{d}{d \tau} \frac{s_{k}^{*}}{H \tau}-\frac{s_{k}^{*}}{H \tau} \frac{d}{d \tau} \frac{s_{k}}{H \tau}=i$.

We require that for $k|\tau| \ll 1$ the canonical fields $\pi_{k} / H \tau$ and $s_{k} / H \tau$ go like $e^{-i k \tau}$, which implies that the mode functions are

$$
\pi_{k}=\frac{H}{\sqrt{2 k}}\left(i \tau+\frac{1}{k}\right) e^{-i k \tau}
$$

and

$$
s_{k}=\frac{H(-k \tau)^{3 / 2} \pi^{1 / 2}}{2 k^{3 / 2}} H_{\alpha}^{(2)}(k \tau)
$$

where

$$
\alpha=\left(\frac{9}{4}-\frac{m^{2}}{H^{2}}\right)^{1 / 2}
$$

and $H_{\alpha}^{(2)}$ is the second Hankel function with index $\alpha$. We assume that the state of the Universe during inflation is the vacuum state $|0\rangle$, defined as

$$
a_{\vec{k}}|0\rangle=b_{\vec{k}}|0\rangle=0, \quad\langle 0 \mid 0\rangle=1
$$

This vacuum state is usually called the Bunch-Davies vacuum [15].

Throughout this paper we focus on the region where $m^{2} / H^{2}$ is small compared with unity. For $|k \tau| \ll 1$,

$$
s_{k}(\eta)=\frac{H \pi^{1 / 2}}{2 k^{3 / 2}}(-k \tau)^{\nu} a_{0}
$$

where

$$
\nu=3 / 2-\alpha \simeq m^{2} /\left(3 H^{2}\right)
$$

and

$$
a_{0}=i \frac{2^{3 / 2-\nu} \Gamma(3 / 2-\nu)}{\pi} \simeq \frac{2^{1 / 2} i}{\pi^{1 / 2}}
$$

We are interested in the multipoint correlation functions of the curvature perturbation far outside the horizon $(\tau \simeq 0)$, so we need to evaluate $\left\langle\zeta\left(\vec{x}_{1}, 0\right) \cdots \zeta\left(\vec{x}_{n}, 0\right)\right\rangle$. This can be calculated using [16]

$$
\begin{aligned}
\langle\mathcal{O}(0)\rangle= & \sum_{N} i^{N} \int_{-\infty}^{0} d \tau_{N} \int_{-\infty}^{\tau_{N}} d \tau_{N-1} \cdots \\
& \times \int_{-\infty}^{\tau_{2}} d \tau_{1}\left\langle\left[ H_{\mathrm{int}}^{I}\left(\tau_{1}\right),\left[H_{\mathrm{int}}^{I}\left(\tau_{1}\right), \cdots\right.\right.\right. \\
& \left.\left.\left.\times H_{\mathrm{int}}^{I}\left(\tau_{N}\right), \mathcal{O}^{I}(0)\right] \cdots\right]\right\rangle
\end{aligned}
$$

where the superscript $I$ stands for the interaction picture.

\section{GENERAL FORM OF THE THREE- AND FOUR-POINT CORRELATION FUNCTIONS OF $\zeta$}

\section{A. Three-point correlation function}

There are two diagrams generating one-loop contributions to the three-point function of $\pi$, which are shown in Fig. 1. We will show that with the saturation of the current limits of $f_{\mathrm{NL}}$ and $\tau_{\mathrm{NL}}$ the contribution from Fig. 1(a) is much larger than that from Fig. 1(b). The calculation of the resulting bispectrum is performed in Appendix A, and we find that for $m / H \ll 1$

$$
\begin{aligned}
\mathcal{B}_{\zeta}\left(\vec{k}_{1}, \vec{k}_{2}, \vec{k}_{3}\right)= & \frac{\pi\left|a_{0}\right|^{6} H^{6}}{128 \Lambda^{6}} \frac{1}{(2 \nu)^{4}} \times\left\{\frac{\max \left(k_{1}, k_{3}\right)^{-2 \nu} k_{\max }^{-2 \nu}}{k_{1}^{3-2 \nu} k_{3}^{3-2 \nu}}\right. \\
& +\frac{\max \left(k_{2}, k_{3}\right)^{-2 \nu} k_{\max }^{-2 \nu}}{k_{2}^{3-2 \nu} k_{3}^{3-2 \nu}} \\
& \left.+\frac{\max \left(k_{1}, k_{2}\right)^{-2 \nu} k_{\max }^{-2 \nu}}{k_{1}^{3-2 \nu} k_{2}^{3-2 \nu}}\right\}
\end{aligned}
$$

This is the general leading-order expression for the bispectrum of $\zeta$.

The factor $\left(k_{i} / k_{j}\right)^{\nu} \simeq 1+\nu \ln \left(k_{i} / k_{j}\right)+\cdots$, so such factors can be set to unity when all of the ratios of the $k$ 's are not very small or large. Then,

$\mathcal{B}_{\zeta}\left(\vec{k}_{1}, \vec{k}_{2}, \vec{k}_{3}\right) \simeq \frac{\pi\left|a_{0}\right|^{6} H^{6}}{128 \Lambda^{6}} \frac{1}{(2 \nu)^{4}} \times\left\{\frac{1}{k_{1}^{3} k_{3}^{3}}+\frac{1}{k_{2}^{3} k_{3}^{3}}+\frac{1}{k_{1}^{3} k_{2}^{3}}\right\}$.

This is the form that the bispectrum has in local nonGaussianity.

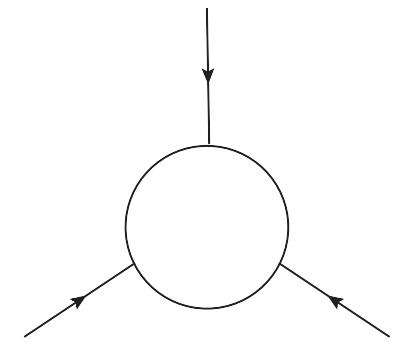

(a)

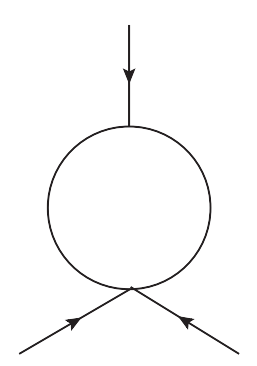

(b)
FIG. 1. One-loop diagrams for three-point functions of $\pi$. 


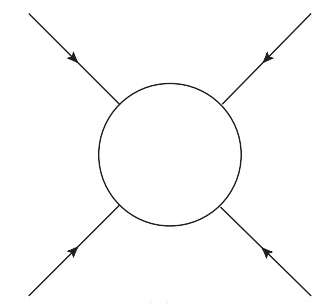

(a)

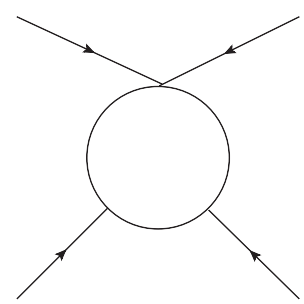

(b)

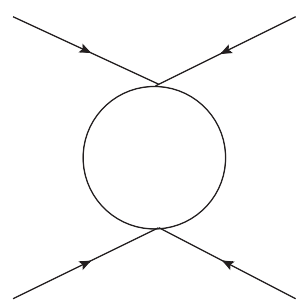

(c)

FIG. 2. One-loop diagrams for four-point functions of $\pi$.

Next we consider the squeezed region where $k \equiv k_{1} \simeq k_{2} \gg k_{3} \equiv q$. It is easy to see that in this limit the bispectrum goes to

$$
\mathcal{B}_{\zeta}\left(\vec{k}_{1}, \vec{k}_{2}, \vec{k}_{3}\right) \simeq \frac{\pi H^{6}\left|a_{0}\right|^{6}}{64 \Lambda^{6}} \frac{1}{(2 \nu)^{4}} \frac{1}{k^{3+2 \nu} q^{3-2 \nu}} .
$$

This differs from what one gets from local non-Gaussianity by a factor of $(q / k)^{2 \nu}$.

\section{B. Four-point correlation function}

There are three Feynman diagrams contributing to the one-loop four-point function of $\zeta$, which are shown in Fig. 2. We will show that with the saturation of the current limits of $f_{\mathrm{NL}}$ and $\tau_{\mathrm{NL}}$ the contribution from Fig. 2(a) dominates. In Appendix B we find that the contribution from Fig. 2(a) is

$$
\begin{aligned}
\mathcal{B}_{\zeta}\left(\vec{k}_{1}, \vec{k}_{2}, \vec{k}_{3}, \vec{k}_{4}\right)= & \frac{\pi^{2} H^{8}\left|a_{0}\right|^{8}}{1024 \Lambda^{8}}\left(\frac{1}{2 \nu}\right)^{5} \times \frac{\min \left[\max \left(k_{1}, k_{2},\left|\vec{k}_{1}+\vec{k}_{2}\right|\right), \max \left(k_{3}, k_{4},\left|\vec{k}_{3}+\vec{k}_{4}\right|\right)\right]^{-2 \nu} \max \left(k_{1}, k_{2}, k_{3}, k_{4},\left|\vec{k}_{1}+\vec{k}_{2}\right|\right)^{-2 \nu}}{k_{1}^{3} k_{4}^{3}\left|\vec{k}_{1}+\vec{k}_{2}\right|^{3-2 \nu} \min \left(k_{1}, k_{4},\left|\vec{k}_{1}+\vec{k}_{2}\right|\right)^{-2 \nu}} \\
& +\operatorname{per}\left(k_{1}, k_{2}, k_{3}, k_{4}\right) .
\end{aligned}
$$

Provided none of the ratios of the $k$ 's or partial sums of the $k$ 's are anomalously large, this has the same form as $\tau_{N L}$ $\left(g_{N L}=0\right)$ non-Gaussianity,

$$
\begin{aligned}
\mathcal{B}_{\zeta}\left(\vec{k}_{1}, \vec{k}_{2}, \vec{k}_{3}, \vec{k}_{4}\right) \simeq & \frac{\pi^{2} H^{8}\left|a_{0}\right|^{8}}{1024 \Lambda^{8}}\left(\frac{1}{2 \nu}\right)^{5} \\
& \times\left(\frac{1}{k_{1}^{3} k_{4}^{3}\left|\vec{k}_{1}+\vec{k}_{2}\right|^{3}}+\operatorname{per}\left(k_{1}, k_{2}, k_{3}, k_{4}\right)\right) .
\end{aligned}
$$

In the compressed region where $k_{1} \simeq k_{2} \gg\left|\vec{k}_{1}+\vec{k}_{2}\right| \equiv q$ and $k_{3} \simeq k_{4} \gg q$, it simplifies to

$$
\mathcal{B}\left(\vec{k}_{1}, \vec{k}_{2}, \vec{k}_{3}, \vec{k}_{4}\right) \simeq \frac{\pi^{2} H^{8}\left|a_{0}\right|^{8}}{128 \Lambda^{8}}\left(\frac{1}{2 \nu}\right)^{5} \frac{1}{k_{1}^{3+2 \nu}} \frac{1}{k_{3}^{3+2 \nu}} \frac{1}{q^{3-4 \nu}}
$$

The scaling of the four-point curvature fluctuation in the compressed limit $\left(\sim q^{-3+4 \nu}\right)$ can be read off from the scaling dimension of the operator $s^{2}$ since this determines the form of its two-point correlation evaluated on the boundary $\tau=0$ of de Sitter space. [17].

\section{CMB CONSTRAINTS}

In the previous section it was noted that for $\nu \ll 1$, the bispectrum of this model is the same as the local nonGaussianity model (for typical wave vectors). Therefore, we can use the observed limit of $f_{\mathrm{NL}}^{\text {local }}$ from the CMB observations of the non-Gaussianity to estimate the constraint on this model. In the limit where the bispectrum reduces to local non-Gaussianity,

$$
\begin{aligned}
f_{\mathrm{NL}}^{\text {local }} & =\frac{5}{6}\left(\frac{\mathcal{B}_{\zeta}\left(k_{1}, k_{2}, k_{3}\right)}{P_{\zeta}\left(k_{1}\right) P_{\zeta}\left(k_{2}\right)+P_{\zeta}\left(k_{1}\right) P_{\zeta}\left(k_{3}\right)+P_{\zeta}\left(k_{2}\right) P_{\zeta}\left(k_{3}\right)}\right) \\
& \simeq \frac{5}{6} \frac{1}{2^{9} \pi^{3}}\left(\frac{H}{\Lambda}\right)^{6}\left|a_{0}\right|^{6}\left(\Delta_{\zeta}^{2}\right)^{-2}\left(\frac{1}{2 \nu}\right)^{4},
\end{aligned}
$$

where

$$
\Delta_{\zeta}^{2} \equiv \frac{1}{(2 \pi)^{2}} \frac{H^{4}}{\dot{\phi}_{0}^{2}} \simeq 2.14 \times 10^{-9}
$$

The $2 \sigma$ constraint [13] on $f_{\mathrm{NL}}^{\text {local }}$ is about $\left|f_{\mathrm{NL}}^{\text {local }}\right|<10$. Therefore, we have

$$
\frac{H}{\Lambda}\left(\frac{1}{2 \nu}\right)^{2 / 3}<0.012
$$


Similarly, the trispectrum $\tau_{\mathrm{NL}}$ can be estimated as

$$
\tau_{\mathrm{NL}} \simeq \frac{H^{8}\left|a_{0}\right|^{8}}{4096 \pi^{4} \Lambda^{8}}\left(\frac{1}{2 \nu}\right)^{5}\left(\Delta_{\zeta}^{2}\right)^{-3}
$$

The constraint on $\tau_{\mathrm{NL}}$ from the Planck observations of the CMB spectrum [11] is

$$
\tau_{\mathrm{NL}}<2800
$$

which implies that

$$
\frac{H}{\Lambda}\left(\frac{1}{2 \nu}\right)^{5 / 8}<0.0095
$$

We can use these constraints to estimate the importance of Fig. 2(b) in comparison with Fig. 2(a) for the four-point correlation. It is straightforward to see that

$$
\frac{\operatorname{diagram} 2(\mathrm{a})}{\operatorname{diagram} 2(\mathrm{~b})} \sim \frac{\dot{\phi}_{0}^{2}}{H^{4}} \frac{H^{2}}{\Lambda^{2}} \frac{1}{\nu}
$$

Treating Eq. (4.6) as an equality, this becomes

$$
\frac{\operatorname{diagram} 2(\mathrm{a})}{\operatorname{diagram} 2(\mathrm{~b})} \sim 10^{-4} \frac{\dot{\phi}_{0}^{2}}{H^{4}} \nu^{1 / 4},
$$

which is much larger than unity unless $\nu$ is exceptionally small. ${ }^{3}$ Similar conclusions hold for Fig. 2(c) and for other correlation functions.

The constraints from $f_{\mathrm{NL}}^{\text {local }}$ and $\tau_{\mathrm{NL}}$ are shown in Fig. 3, where one can see that the constraint from $\tau_{\mathrm{NL}}$ is always stronger. This result is in contrast to many other models, where the constraint from $f_{\mathrm{NL}}$ is stronger. The main reason is the following. In the models where there are tree-level contributions to non-Gaussianity, $f_{\mathrm{NL}}$ is usually proportional to a single power of the small coupling of the new interaction and $\tau_{\mathrm{NL}}$ is proportional to the square of it, whereas in this model $f_{\mathrm{NL}}$ is proportional to the third power of the coupling and $\tau_{\mathrm{NL}}$ is proportional to the fourth power of it. Therefore, in this model $\tau_{\mathrm{NL}}$ is relatively more important.

Since there is some small-time dependence in $\dot{\phi}_{0}$, Eq. (2.2) implies that the $s$ mass depends on time,

$$
\frac{d \ln m^{2}}{H d t} \simeq \frac{1}{24 \pi^{2} \Delta_{\zeta}^{2}}\left(\frac{H^{2}}{\Lambda^{2}}\right) \frac{\eta}{\nu} .
$$

\footnotetext{
${ }^{3}$ Although we have only calculated our results to leading order in $1 / \nu, \nu \sim 0.1$ should be small enough for these results to be a reasonable approximation.
}

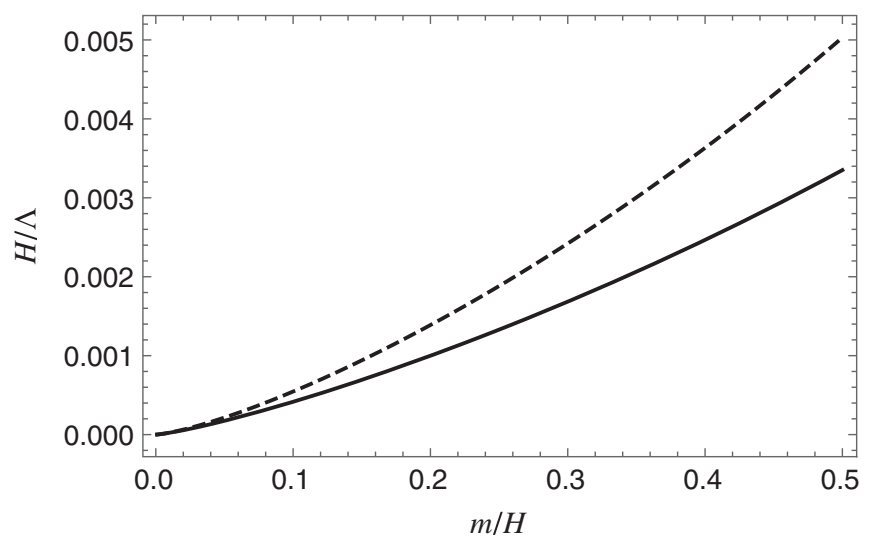

FIG. 3. The dashed and solid curves show the upper limits on $H / \Lambda$ as a function of $m / H$ from $f_{\mathrm{NL}}$ and $\tau_{\mathrm{NL}}$.

The slow-roll parameter $\eta$ must be small enough so that this time dependence can be neglected in our computations. Indeed, our computations of the leading contributions in the three- and four-point correlation functions in Eqs. (3.2) and (3.6) are based on the infrared enhancement of the integral

$$
\int_{-1}^{0} \frac{d(k \tau)}{(-(k \tau))^{1-\nu}}=\frac{1}{\nu}
$$

The main support of this integral is in the region $k \tau \in\left(-1,-e^{-1 / \nu}\right)$. The change of $m^{2}$ in this region can be estimated as

$$
\Delta \ln \left(m^{2} / H^{2}\right) \approx \frac{1}{24 \pi^{2} \Delta_{\zeta}^{2}}\left(\frac{H^{2}}{\Lambda^{2}}\right) \frac{\eta}{\nu^{2}}
$$

For the model to be perturbative, we usually require $\dot{\phi}_{0} / \Lambda^{2} \ll 1$ since this is the effective coupling of $\dot{\pi}$ to two $s$ fields. Together with Eq. (4.2), this implies that

$$
\Delta \ln \left(m^{2} / H^{2}\right) \ll \frac{1}{6} \frac{|\eta|}{\nu^{2}}
$$

Combining this with the requirement of perturbativity, the conservative requirement of $\eta$ can be written as

$$
|\eta| \lesssim \nu^{2}
$$

Similarly, the validity of our leading-order estimation also requires $H$ to not change much in the main support of the integral in Eq. (4.10), which results in

$$
\left|\frac{\Delta H}{H}\right| \approx \epsilon \nu^{-1} \ll 1,
$$


where $\epsilon \equiv-\dot{H} / H^{2}$ is the first slow-roll parameter measuring the change of the Hubble parameter during inflation. Then the requirement for our estimates to be valid is

$$
\epsilon \ll \nu
$$

If the change of $\nu$ cannot be neglected, the integral in Eq. (4.10) can be estimated as

$$
\int_{-1}^{0} \frac{d(k \tau)}{(-(k \tau))^{1-\nu(\tau)}}
$$

and if the result is much larger than unity, we can still use the leading-order approximation but in the $\tau$ integrals we need to take into account the $\tau$ dependence in $H, m$, and $\nu$.

In Appendix $\mathrm{C}$ we calculate the one-loop correction to $\Delta_{\zeta}^{2}$ and find that

$$
\delta \Delta_{\zeta}^{2}=\frac{H^{4}\left|a_{0}\right|^{4}}{512 \pi^{2} \nu^{3} \Lambda^{4}}
$$

The constraints from $\tau_{\mathrm{NL}}$ and $f_{\mathrm{NL}}$ are

$\delta \Delta_{\zeta}^{2}<\min \left[6 \times 10^{-12} \times\left(\frac{1}{2 \nu}\right)^{1 / 2}, 1.3 \times 10^{-11} \times\left(\frac{1}{2 \nu}\right)^{1 / 3}\right]$,

which are much smaller than the observed value given in Eq. (4.2) unless $\nu$ is exceptionally small (see footnote 3 ). So the two-point correlation is dominated by the tree-level result.

\section{CONCLUDING REMARKS}

We have examined the primordial curvature perturbations in an inflationary cosmology where the inflaton $\phi$ couples to an additional scalar field $s$ with mass $m \ll H$ through the nonrenormalizable interaction $g^{\mu \nu} \partial_{\mu} \phi \partial_{\nu} \phi s^{2} / 4 \Lambda^{2}$. In this model primordial non-Gaussianities arise from quantum loop diagrams (with a virtual $s$ in the loop) in de Sitter space. The primordial curvature fluctuation bispectrum and trispectrum in this model were computed. Typically, they have the form of local non-Gaussianity and $\tau_{N L}$ nonGaussianity, respectively, although in squeezed and compressed limits there are deviations from those forms.

Given the work in this paper, it is not difficult to deduce the form of the $n>4$ curvature perturbations. In the situation where all of the wave vectors and their partial sums are of order $k$,

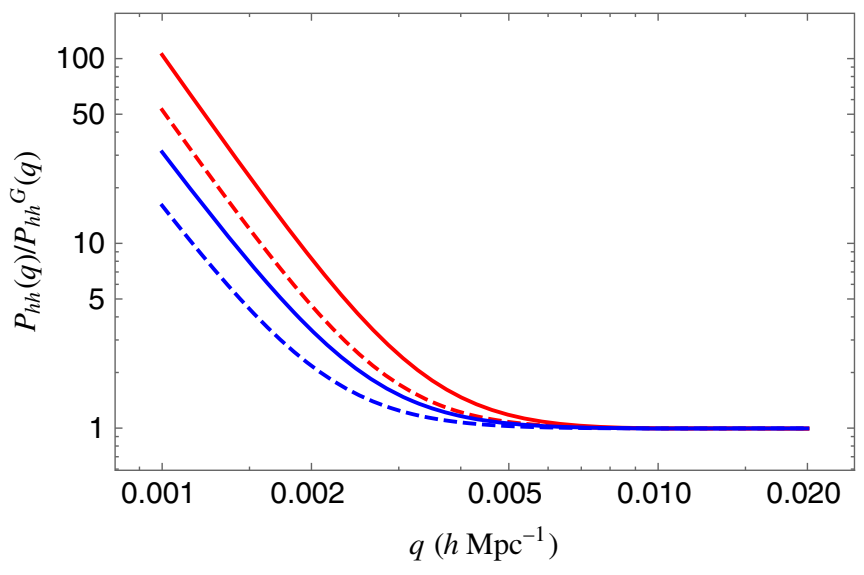

FIG. 4. The red solid and dashed curves are for $\nu=0.05$ with $\tau_{\mathrm{NL}}=2800$ and 1400, respectively, and the blue solid and dashed curves are for $\nu=0.1$ with the same choices of $\tau_{\mathrm{NL}}$.

$$
B_{\zeta}\left(\vec{k}_{1}, \ldots, \vec{k}_{n}\right) \sim\left(\frac{H}{\Lambda}\right)^{2 n}\left(\frac{1}{2 \nu}\right)^{n+1} \frac{1}{k^{3 n-3}},
$$

and in the limit where a single partial sum of wave vectors $\left|\vec{k}_{1}+\cdots+\vec{k}_{j}\right|=q \ll k$ it becomes

$B_{\zeta}\left(\vec{k}_{1}, \ldots, \vec{k}_{n}\right) \sim\left(\frac{H}{\Lambda}\right)^{2 n}\left(\frac{1}{2 \nu}\right)^{n+1} \frac{1}{k^{3 n-6+4 \nu}} \frac{1}{q^{3-4 \nu}}$.

In this model, due to the IR behavior of the compressed trispectrum (3.6), the long-distance behavior of the power spectrum for fluctuations in the galaxy number density is enhanced by a factor of $q^{4-4 \nu}$ compared to the HarrisonZel'dovich spectrum (which goes, apart from a small tilt, as $q$ ). Following the same procedure as described in Refs. $[7,10]$ we estimate this power spectrum, and its ratio with the leading Harrison-Zel'dovich contribution is shown in Fig. 4 using the curvature bispectrum and trispectrum calculated in this paper. One can see that if the current constraint from $\tau_{\mathrm{NL}}$ is saturated, the power spectrum of the galactic halo distribution can differ significantly from the Harrison-Zel'dovich spectrum at $q \sim h /(250(\mathrm{Mpc}))$. These deviations from what Gaussian primordial fluctuations would give can become very large on scales around $500 h^{-1} \mathrm{Mpc}$.

\section{ACKNOWLEDGMENTS}

H. A. is supported by the Recruitment Program for Young Professionals of the 1000 Talented Plan and the Tsinghua University Initiative Scientific Research Program. M. B. W. is supported by the DOE Grant No. DE-SC0011632 and the Walter Burke Institute for Theoretical Physics. 


\section{APPENDIX A: THE BISPECTRUM}

The contribution from Fig. 1(a) can be written as

$$
\begin{aligned}
\mathcal{A}_{3}^{(a)}\left(\vec{x}_{1}, \vec{x}_{2}, \vec{x}_{3}\right) \equiv & \left\langle\zeta\left(\vec{x}_{1}, 0\right) \zeta\left(\vec{x}_{1}, 0\right) \zeta\left(\vec{x}_{3}, 0\right)\right\rangle(a) \\
= & \frac{i}{8 \Lambda^{6} H^{6}} \int_{-\infty}^{0} \frac{d \tau_{3}}{\tau_{3}^{3}} \int_{-\infty}^{\tau_{3}} \frac{d \tau_{2}}{\tau_{2}^{3}} \int_{-\infty}^{\tau_{2}} \frac{d \tau_{1}}{\tau_{1}^{3}} \int d^{3} y_{1} d^{3} y_{2} d^{3} y_{3} \\
& \times\left\langle\left[\pi^{\prime}\left(\tau_{1}, \vec{y}_{1}\right) s^{2}\left(\tau_{1}, \vec{y}_{1}\right),\left[\pi^{\prime}\left(\tau_{2}, \vec{y}_{2}\right) s^{2}\left(\tau_{2}, \vec{y}_{2}\right),\left[\pi^{\prime}\left(\tau_{3}, \vec{y}_{3}\right) s^{2}\left(\tau_{3}, \vec{y}_{3}\right), \pi\left(0, \vec{x}_{1}\right) \pi\left(0, \vec{x}_{2}\right) \pi\left(0, \vec{x}_{3}\right)\right]\right]\right]\right\rangle .
\end{aligned}
$$

Here a ' denotes a derivative with respect to the conformal time $\tau$. The calculation can be done step by step (from inside to outside of the commutation relations) in the following way:

$\left[\pi^{\prime}\left(\tau_{3}, \vec{y}_{3}\right) s^{2}\left(\tau_{3}, \vec{y}_{3}\right), \pi\left(0, \vec{x}_{1}\right) \pi\left(0, \vec{x}_{2}\right) \pi\left(0, \vec{x}_{3}\right)\right]=\left[\pi^{\prime}\left(\tau_{3}, \vec{y}_{3}\right), \pi\left(0, \vec{x}_{1}\right)\right] s^{2}\left(\tau_{3}, \vec{y}_{3}\right) \pi\left(0, \vec{x}_{2}\right) \pi\left(0, \vec{x}_{3}\right)+\left(x_{1} \leftrightarrow x_{2}\right)+\left(x_{1} \leftrightarrow x_{3}\right)$.

Note that $\left[\pi^{\prime}\left(\tau_{3}, \vec{y}_{3}\right), \pi\left(0, \vec{x}_{1}\right)\right]$ is

$$
\left[\pi^{\prime}\left(\tau_{3}, \vec{y}_{3}\right), \pi\left(0, \vec{x}_{1}\right)\right]=\left\langle\left[\pi^{\prime}\left(\tau_{3}, \vec{y}_{3}\right), \pi\left(0, \vec{x}_{1}\right)\right]\right\rangle=2 i \operatorname{Im}\left\langle\pi^{\prime}\left(\tau_{3}, \vec{y}_{3}\right) \pi\left(0, \vec{x}_{1}\right)\right\rangle
$$

Therefore, we have

$$
\begin{aligned}
& {\left[\pi^{\prime}\left(\tau_{2}, \vec{y}_{2}\right) s^{2}\left(\tau_{2}, \vec{y}_{2}\right),\left[\pi^{\prime}\left(\tau_{3}, \vec{y}_{3}\right) s^{2}\left(\tau_{3}, \vec{y}_{3}\right), \pi\left(0, \vec{x}_{1}\right) \pi\left(0, \vec{x}_{2}\right) \pi\left(0, \vec{x}_{3}\right)\right]\right]} \\
& \quad=2 i \operatorname{Im}\left\langle\pi^{\prime}\left(\tau_{3}, \vec{y}_{3}\right) \pi\left(0, \vec{x}_{1}\right)\right\rangle\left[\pi^{\prime}\left(\tau_{2}, \vec{y}_{2}\right) s^{2}\left(\tau_{2}, \vec{y}_{2}\right), s^{2}\left(\tau_{3}, \vec{y}_{3}\right) \pi\left(0, \vec{x}_{2}\right) \pi\left(0, \vec{x}_{3}\right)\right] .
\end{aligned}
$$

From the structure of Fig. 1(a) we find that one $s$ at $\left(\tau_{2}, \vec{y}_{2}\right)$ should contract with one $s$ at $\left(\tau_{3}, \vec{y}_{3}\right)$, and the other $s$ 's should contract with the $s^{2}$ at $\left(\tau_{1}, \vec{y}_{1}\right)$. Therefore, we have

$$
\begin{aligned}
& {\left[\pi^{\prime}\left(\tau_{2}, \vec{y}_{2}\right) s^{2}\left(\tau_{2}, \vec{y}_{2}\right), s^{2}\left(\tau_{3}, \vec{y}_{3}\right) \pi\left(0, \vec{x}_{2}\right) \pi\left(0, \vec{x}_{3}\right)\right]_{\mathrm{Fig} .1(\mathrm{a})}} \\
& \quad=4\left\langle\left[\pi^{\prime}\left(\tau_{2}, \vec{y}_{2}\right) s\left(\tau_{2}, \vec{y}_{2}\right), s\left(\tau_{3}, \vec{y}_{3}\right) \pi\left(0, \vec{x}_{2}\right)\right]\right\rangle s\left(\tau_{2}, \vec{y}_{2}\right) s\left(\tau_{3}, \vec{y}_{3}\right) \pi\left(0, \vec{x}_{3}\right)+\left(x_{2} \leftrightarrow x_{3}\right) \\
& \quad=8 i \operatorname{Im}\left[\left\langle\pi^{\prime}\left(\tau_{2}, \vec{y}_{2}\right) \pi\left(0, \vec{x}_{2}\right)\right\rangle\left\langle s\left(\tau_{2}, \vec{y}_{2}\right) s\left(\tau_{3}, \vec{y}_{3}\right)\right\rangle\right] s\left(\tau_{2}, \vec{y}_{2}\right) s\left(\tau_{3}, \vec{y}_{3}\right) \pi\left(0, \vec{x}_{3}\right)+\left(x_{2} \leftrightarrow x_{3}\right) .
\end{aligned}
$$

Therefore, we have for the contribution from Fig. 1(a)

$$
\begin{aligned}
\left\langle\left[\pi^{\prime}\left(\tau_{1}, \vec{y}_{1}\right) s^{2}\left(\tau_{1}, \vec{y}_{1}\right),\left[\pi^{\prime}\left(\tau_{2}, \vec{y}_{2}\right) s^{2}\left(\tau_{2}, \vec{y}_{2}\right),\left[\pi^{\prime}\left(\tau_{3}, \vec{y}_{3}\right) s^{2}\left(\tau_{3}, \vec{y}_{3}\right), \pi\left(0, \vec{x}_{1}\right) \pi\left(0, \vec{x}_{2}\right) \pi\left(0, \vec{x}_{3}\right)\right]\right]\right]\right\rangle \\
=-16 \operatorname{Im}\left\langle\pi^{\prime}\left(\tau_{3}, \vec{y}_{3}\right) \pi\left(0, \vec{x}_{1}\right)\right\rangle \operatorname{Im}\left[\left\langle\pi^{\prime}\left(\tau_{2}, \vec{y}_{2}\right) \pi\left(0, \vec{x}_{2}\right)\right\rangle\left\langle s\left(\tau_{2}, \vec{y}_{2}\right) s\left(\tau_{3}, \vec{y}_{3}\right)\right\rangle\right] \\
\quad \times\left\langle\left[\pi^{\prime}\left(\tau_{1}, \vec{y}_{1}\right) s^{2}\left(\tau_{1}, \vec{y}_{1}\right), s\left(\tau_{2}, \vec{y}_{2}\right) s\left(\tau_{3}, \vec{y}_{3}\right) \pi\left(0, \vec{x}_{3}\right)\right]\right\rangle+\operatorname{per}\left(\mathrm{x}_{1}, \mathrm{x}_{2}, \mathrm{x}_{3}\right) \\
=-64 i \operatorname{Im}\left\langle\pi^{\prime}\left(\tau_{3}, \vec{y}_{3}\right) \pi\left(0, \vec{x}_{1}\right)\right\rangle \operatorname{Im}\left[\left\langle\pi^{\prime}\left(\tau_{2}, \vec{y}_{2}\right) \pi\left(0, \vec{x}_{2}\right)\right\rangle\left\langle s\left(\tau_{2}, \vec{y}_{2}\right) s\left(\tau_{3}, \vec{y}_{3}\right)\right\rangle\right] \\
\quad \times \operatorname{Im}\left[\left\langle\pi^{\prime}\left(\tau_{1}, \vec{y}_{1}\right) \pi\left(0, \vec{x}_{3}\right)\right\rangle\left\langle s\left(\tau_{1}, \vec{y}_{1}\right) s\left(\tau_{2}, \vec{y}_{2}\right)\right\rangle\left\langle s\left(\tau_{1}, \vec{y}_{1}\right) s\left(\tau_{3}, \vec{y}_{3}\right)\right\rangle\right]+\operatorname{per}\left(\mathrm{x}_{1}, \mathrm{x}_{2}, \mathrm{x}_{3}\right) .
\end{aligned}
$$

Now we take the second factor as an example,

$$
\operatorname{Im}\left[\left\langle\pi^{\prime}\left(\tau_{2}, \vec{y}_{2}\right) \pi\left(0, \vec{x}_{2}\right)\right\rangle\left\langle s\left(\tau_{2}, \vec{y}_{2}\right) s\left(\tau_{3}, \vec{y}_{3}\right)\right\rangle\right]=\int \frac{d^{3} p}{(2 \pi)^{3}} \frac{d^{3} q}{(2 \pi)^{3}} \operatorname{Im}\left[\pi_{p}^{\prime}\left(\tau_{2}\right) \pi_{p}^{*}(0) e^{i \vec{p} \cdot\left(\vec{y}_{2}-\vec{x}_{2}\right)} s_{q}\left(\tau_{2}\right) s_{q}^{*}\left(\tau_{3}\right) e^{i \vec{q} \cdot\left(\vec{y}_{2}-\vec{y}_{3}\right)}\right] .
$$

Since the mode functions $\pi_{p}$ and $s_{q}$ are even functions of $\vec{p}$ and $\vec{q}$ we can move the exponentials outside. Therefore, we have

$$
\operatorname{Im}\left[\left\langle\pi^{\prime}\left(\tau_{2}, \vec{y}_{2}\right) \pi\left(0, \vec{x}_{2}\right)\right\rangle\left\langle s\left(\tau_{2}, \vec{y}_{2}\right) s\left(\tau_{3}, \vec{y}_{3}\right)\right\rangle\right]=\int \frac{d^{3} p}{(2 \pi)^{3}} \frac{d^{3} q}{(2 \pi)^{3}} e^{i \vec{p} \cdot\left(\vec{y}_{2}-\vec{x}_{2}\right)+i \vec{q} \cdot\left(\vec{y}_{2}-\vec{y}_{3}\right)} \operatorname{Im}\left[\pi_{p}^{\prime}\left(\tau_{2}\right) \pi_{p}^{*}(0) s_{q}\left(\tau_{2}\right) s_{q}^{*}\left(\tau_{3}\right)\right] .
$$


Therefore, we have

$$
\begin{aligned}
\mathcal{A}_{3}^{(a)}\left(\vec{x}_{1}, \vec{x}_{2}, \vec{x}_{3}\right)= & \frac{8}{\Lambda^{6} H^{6}} \int_{-\infty}^{0} \frac{d \tau_{3}}{\tau_{3}^{3}} \int_{-\infty}^{\tau_{3}} \frac{d \tau_{2}}{\tau_{2}^{3}} \int_{-\infty}^{\tau_{2}} \frac{d \tau_{1}}{\tau_{1}^{3}} \int \frac{d^{3} k_{1}}{(2 \pi)^{3}} \frac{d^{3} k_{2}}{(2 \pi)^{3}} \frac{d^{3} k_{3}}{(2 \pi)^{3}}(2 \pi)^{3} \delta^{3}\left(\vec{k}_{1}+\vec{k}_{2}+\vec{k}_{3}\right) e^{i\left(\vec{k}_{1} \cdot x_{1}+\vec{k}_{2} \cdot x_{2}+\vec{k}_{3} \cdot x_{3}\right)} \\
& \times \int \frac{d^{3} p}{(2 \pi)^{3}} \times \operatorname{Im}\left[\pi_{k_{1}}^{\prime}\left(\tau_{3}\right) \pi_{k_{1}}^{*}(0)\right] \operatorname{Im}\left[\pi_{k_{2}}^{\prime}\left(\tau_{2}\right) \pi_{k_{2}}^{*}(0) s_{\left|\vec{p}+\vec{k}_{1}\right|}\left(\tau_{2}\right) s_{\left|\vec{p}+\vec{k}_{1}\right|}^{*}\left(\tau_{3}\right)\right] \\
& \times \operatorname{Im}\left[\pi_{k_{3}}^{\prime}\left(\tau_{1}\right) \pi_{k_{3}}^{*}(0) s_{\left|\vec{p}-\vec{k}_{3}\right|}\left(\tau_{1}\right) s_{\left|\vec{p}-\vec{k}_{3}\right|}^{*}\left(\tau_{2}\right) s_{p}\left(\tau_{1}\right) s_{p}^{*}\left(\tau_{3}\right)\right]+\operatorname{per}\left(x_{1}, x_{2}, x_{3}\right) .
\end{aligned}
$$

The corresponding part of the bispectrum of $\zeta, \mathcal{B}_{\zeta}^{(a)}\left(\vec{k}_{1}, \vec{k}_{2}, \vec{k}_{3}\right)$, is defined as

$$
\mathcal{A}_{3}^{(a)}\left(\vec{x}_{1}, \vec{x}_{2}, \vec{x}_{3}\right)=\int \frac{d^{3} k_{1}}{(2 \pi)^{3}} \frac{d^{3} k_{2}}{(2 \pi)^{3}} \frac{d^{3} k_{3}}{(2 \pi)^{3}} e^{i\left(\vec{k}_{1} \cdot \vec{x}_{1}+\vec{k}_{2} \cdot \vec{x}_{2}+\vec{k}_{3} \cdot \vec{x}_{3}\right)}(2 \pi)^{3} \delta^{3}\left(\vec{k}_{1}+\vec{k}_{2}+\vec{k}_{3}\right) \mathcal{B}_{\zeta}^{(a)}\left(\vec{k}_{1}, \vec{k}_{2}, \vec{k}_{3}\right)
$$

and from Eq. (A9) it is easy to see that

$$
\begin{aligned}
\mathcal{B}_{\zeta}^{(a)}\left(\vec{k}_{1}, \vec{k}_{2}, \vec{k}_{3}\right)= & \frac{8}{\Lambda^{6} H^{6}} \int_{-\infty}^{0} \frac{d \tau_{3}}{\tau_{3}^{3}} \int_{-\infty}^{\tau_{3}} \frac{d \tau_{2}}{\tau_{2}^{3}} \int_{-\infty}^{\tau_{2}} \frac{d \tau_{1}}{\tau_{1}^{3}} \int \frac{d^{3} p}{(2 \pi)^{3}} \operatorname{Im}\left[\pi_{k_{1}}^{\prime}\left(\tau_{3}\right) \pi_{k_{1}}^{*}(0)\right] \operatorname{Im}\left[\pi_{k_{2}}^{\prime}\left(\tau_{2}\right) \pi_{k_{2}}^{*}(0) s_{\left|\vec{p}+\vec{k}_{1}\right|}\left(\tau_{2}\right) s_{\left|\vec{p}+\vec{k}_{1}\right|}^{*}\left(\tau_{3}\right)\right] \\
& \times \operatorname{Im}\left[\pi_{k_{3}}^{\prime}\left(\tau_{1}\right) \pi_{k_{3}}^{*}(0) s_{\left|\vec{p}-\vec{k}_{3}\right|}\left(\tau_{1}\right) s_{\left|\vec{p}-\vec{k}_{3}\right|}^{*}\left(\tau_{2}\right) s_{p}\left(\tau_{1}\right) s_{p}^{*}\left(\tau_{3}\right)\right]+\operatorname{per}\left(k_{1}, k_{2}, k_{3}\right) .
\end{aligned}
$$

In the limit of a massless $s$ one can show that all of the $\tau_{i}$ integrals experience IR divergences. This tells us that in the region where $m / H \ll 1$ the IR contribution dominates the $\tau_{i}$ integrals. Therefore, we can capture the main contribution by Laurent expanding the integrand and calculating the contributions from the leading term.

The Hankel function $H_{\alpha}^{(2)}(k \tau)$ in general can be expanded into two series,

$$
H_{\alpha}^{(2)}(k \tau)=\sum_{n=0}^{\infty} a_{n}(-k \tau)^{-\alpha+n}+\sum_{n=0}^{\infty} b_{n}(-k \tau)^{2 \alpha+n}
$$

The important property is that the coefficients in each series share the same phase. Then, after some straightforward calculations the leading contribution of the bispectrum of $\zeta$ can be written as

$$
\begin{aligned}
\mathcal{B}_{\zeta}^{(a)}\left(\vec{k}_{1}, \vec{k}_{2}, \vec{k}_{3}\right) \simeq & \frac{\pi^{3} H^{6}\left|a_{0}\right|^{6}}{64 \Lambda^{6}} \int \frac{d^{3} p}{(2 \pi)^{3}} \int_{-\Lambda_{3}}^{0} \frac{d \tau_{3}}{\left(-\tau_{3}\right)^{1-2 \nu}} \int_{-\Lambda_{2}}^{\tau_{3}} \frac{d \tau_{2}}{\left(-\tau_{2}\right)^{1-2 \nu}} \int_{-\Lambda_{1}}^{\tau_{2}} \frac{d \tau_{1}}{\left(-\tau_{1}\right)^{1-2 \nu}} \\
& \times \frac{1}{\left|\vec{p}+\vec{k}_{1}\right|^{3-2 \nu}\left|\vec{p}-\vec{k}_{3}\right|^{3-2 \nu} p^{3-2 \nu}}+\operatorname{per}\left(k_{1}, k_{2}, k_{3}\right) .
\end{aligned}
$$

The UV cutoff of each $\tau_{i}$ integral is determined by the point where the integrant starts to oscillate,

$$
\Lambda_{1}=\min \left(k_{3}^{-1},\left|\vec{p}-\vec{k}_{3}\right|^{-1}, p^{-1}\right), \quad \Lambda_{2}=\min \left(k_{2}^{-1},\left|\vec{p}+\vec{k}_{1}\right|^{-1},\left|\vec{p}-\vec{k}_{3}\right|^{-1}\right), \quad \Lambda_{3}=\min \left(k_{1}^{-1},\left|\vec{p}+\vec{k}_{1}\right|^{-1}, p^{-1}\right)
$$

Now we can evaluate the $\tau_{i}$ integrals explicitly, and we have

$$
\mathcal{B}_{\zeta}^{(a)}\left(\vec{k}_{1}, \vec{k}_{2}, \vec{k}_{3}\right)=\frac{\pi^{3} H^{6}\left|a_{0}\right|^{6}}{64 \Lambda^{6}} \frac{1}{(2 \nu)^{3}} \int \frac{d^{3} p}{(2 \pi)^{3}} \frac{\mathcal{I}_{3}\left(\Lambda_{1}, \Lambda_{2}, \Lambda_{3}\right)}{\left|\vec{p}+\vec{k}_{1}\right|^{3-2 \nu}\left|\vec{p}-\vec{k}_{3}\right|^{3-2 \nu} p^{3-2 \nu}}+\operatorname{per}\left(k_{1}, k_{2}, k_{3}\right),
$$

where

$$
\mathcal{I}_{3}\left(\Lambda_{1}, \Lambda_{2}, \Lambda_{3}\right)=\left(\Lambda_{1} \Lambda_{12} \Lambda_{123}\right)^{2 \nu}-\frac{1}{2} \Lambda_{12}^{4 \nu} \Lambda_{123}^{2 \nu}-\frac{1}{2} \Lambda_{1}^{2 \nu} \Lambda_{123}^{4 \nu}+\frac{1}{6} \Lambda_{123}^{6 \nu}
$$


and

$$
\begin{aligned}
\Lambda_{12} & =\min \left[\Lambda_{1}, \Lambda_{2}\right]=\min \left(k_{3}^{-1},\left|\vec{p}-\vec{k}_{3}\right|^{-1}, p^{-1}, k_{2}^{-1},\left|\vec{p}+\vec{k}_{1}\right|^{-1}\right), \\
\Lambda_{123} & =\min \left[\Lambda_{1}, \Lambda_{2}, \Lambda_{3}\right]=\min \left(k_{3}^{-1},\left|\vec{p}-\vec{k}_{3}\right|^{-1}, p^{-1}, k_{2}^{-1},\left|\vec{p}+\vec{k}_{1}\right|^{-1}, k_{1}^{-1}\right) .
\end{aligned}
$$

The $p$ integral is also controlled by the infrared contributions. One can see that the integrant of the $p$ integral has three branch points, and the integral is supported by the domain around these branch points. For example, for the branch point at $p=0$, the integral is mainly supported in the region around $p \lesssim \nu \times \min \left(k_{1}, k_{3}\right)$. Therefore, one can see that the three regions are well separated. Therefore, the leading-order contribution of the integral can be separated into three parts as

$$
\begin{aligned}
\int \frac{d^{3} p}{(2 \pi)^{3}} \frac{\mathcal{I}_{3}\left(\Lambda_{1}, \Lambda_{2}, \Lambda_{3}\right)}{\left|\vec{p}+\vec{k}_{1}\right|^{3-2 \nu}\left|\vec{p}-\vec{k}_{3}\right|^{3-2 \nu} p^{3-2 \nu} \simeq} & \frac{1}{4 \pi^{2} \nu}\left\{\frac{\left.\mathcal{I}_{3}\left(\Lambda_{1}, \Lambda_{2}, \Lambda_{3}\right)\right|_{p \rightarrow 0}}{k_{1}^{3-2 \nu} k_{3}^{3-2 \nu} \min \left(k_{1}, k_{3}\right)^{-2 \nu}}+\frac{\left.\mathcal{I}_{3}\left(\Lambda_{1}, \Lambda_{2}, \Lambda_{3}\right)\right|_{\vec{p} \rightarrow-\vec{k}_{1}}}{k_{1}^{3-2 \nu} k_{2}^{3-2 \nu} \min \left(k_{1}, k_{2}\right)^{-2 \nu}}\right. \\
& \left.+\frac{\left.\mathcal{I}_{3}\left(\Lambda_{1}, \Lambda_{2}, \Lambda_{3}\right)\right|_{\vec{p} \rightarrow \vec{k}_{3}}}{k_{2}^{3-2 \nu} k_{3}^{3-2 \nu} \min \left(k_{2}, k_{3}\right)^{-2 \nu}}\right\} \\
= & \frac{1}{4 \pi^{2} \nu}\left\{\frac{\frac{1}{2} k_{3}^{-2 \nu} k_{\max }^{-4 \nu}-\frac{1}{3} k_{\max }^{-6 \nu}}{k_{1}^{3-2 \nu} k_{3}^{3-2 \nu} \min \left(k_{1}, k_{3}\right)^{-2 \nu}}+\frac{\frac{1}{6} k_{\max }^{-6 \nu}}{k_{1}^{3-2 \nu} k_{2}^{3-2 \nu} \min \left(k_{1}, k_{2}\right)^{-2 \nu}}\right. \\
& \left.+\frac{k_{3}^{-2 \nu} \max \left(k_{2}, k_{3}\right)^{-2 \nu} k_{\max }^{-2 \nu}-\frac{1}{2} \max \left(k_{2}, k_{3}\right)^{-4 \nu} k_{\max }^{-2 \nu}-\frac{1}{2} k_{3}^{-2 \nu} k_{\max }^{-4 \nu}+\frac{1}{6} k_{\max }^{-6 \nu}}{k_{2}^{3-2 \nu} k_{3}^{3-2 \nu} \min \left(k_{2}, k_{3}\right)^{-2 \nu}}\right\},
\end{aligned}
$$

where $k_{\max }$ stands for $\max \left(k_{1}, k_{2}, k_{3}\right)$.

With this permutation [and noting that the diagram in Fig. 1(a) is dominant], after straightforward calculations one can show that the leading contribution to the bispectrum is

$$
\mathcal{B}_{\zeta}\left(\vec{k}_{1}, \vec{k}_{2}, \vec{k}_{3}\right)=\frac{\pi\left|a_{0}\right|^{6} H^{6}}{128 \Lambda^{6}} \frac{1}{(2 \nu)^{4}} \times\left\{\frac{\max \left(k_{1}, k_{3}\right)^{-2 \nu} k_{\max }^{-2 \nu}}{k_{1}^{3-2 \nu} k_{3}^{3-2 \nu}}+\frac{\max \left(k_{2}, k_{3}\right)^{-2 \nu} k_{\max }^{-2 \nu}}{k_{2}^{3-2 \nu} k_{3}^{3-2 \nu}}+\frac{\max \left(k_{1}, k_{2}\right)^{-2 \nu} k_{\max }^{-2 \nu}}{k_{1}^{3-2 \nu} k_{2}^{3-2 \nu}}\right\} .
$$

Equation (A19) gives the general leading-order expression for the bispectrum of $\zeta$ in the region where $m / H \ll 1$.

\section{APPENDIX B: THE TRISPECTRUM}

There are in general three Feynman diagrams contributing to the one-loop four-point function of $\zeta$, which are shown in Fig. 2. Following Eq. (2.17), the contribution from Fig. 2(a) can be written as

$$
\begin{aligned}
\mathcal{A}_{4}^{(a)}\left(\vec{x}_{1}, \vec{x}_{2}, \vec{x}_{3}, \vec{x}_{4}\right) \equiv & \left\langle\zeta\left(\vec{x}_{1}, 0\right) \zeta\left(\vec{x}_{1}, 0\right) \zeta\left(\vec{x}_{3}, 0\right) \zeta\left(\vec{x}_{4}, 0\right)\right\rangle(a) \\
= & \frac{1}{16 \Lambda^{8} H^{8}} \int_{-\infty}^{0} \frac{d \tau_{4}}{\tau_{4}^{3}} \int_{-\infty}^{\tau_{4}} \frac{d \tau_{3}}{\tau_{3}^{3}} \int_{-\infty}^{\tau_{3}} \frac{d \tau_{2}}{\tau_{2}^{3}} \int_{-\infty}^{\tau_{2}} \frac{d \tau_{1}}{\tau_{1}^{3}} \int d^{3} y_{1} d^{3} y_{2} d^{3} y_{3} d^{3} y_{4} \\
& \times\left\langle\left[\pi^{\prime}\left(\tau_{1}, \vec{y}_{1}\right) s^{2}\left(\tau_{1}, \vec{y}_{1}\right),\left[\pi^{\prime}\left(\tau_{2}, \vec{y}_{2}\right) s^{2}\left(\tau_{2}, \vec{y}_{2}\right),\left[\pi^{\prime}\left(\tau_{3}, \vec{y}_{3}\right) s^{2}\left(\tau_{3}, \vec{y}_{3}\right),\left[\pi^{\prime}\left(\tau_{4}, \vec{y}_{4}\right) s^{2}\left(\tau_{4}, \vec{y}_{4}\right),\right.\right.\right.\right.\right. \\
& \left.\left.\left.\left.\left.\times \pi\left(0, \vec{x}_{1}\right) \pi\left(0, \vec{x}_{2}\right) \pi\left(0, \vec{x}_{3}\right) \pi\left(0, \vec{x}_{4}\right)\right]\right]\right]\right]\right\rangle .
\end{aligned}
$$

We follow a similar procedure and calculate the integrant from inside out,

$$
\begin{aligned}
\mathcal{S}_{1} & \equiv\left[\pi^{\prime}\left(\tau_{4}, \vec{y}_{4}\right) s^{2}\left(\tau_{4}, \vec{y}_{4}\right), \pi\left(0, \vec{x}_{1}\right) \pi\left(0, \vec{x}_{2}\right) \pi\left(0, \vec{x}_{3}\right) \pi\left(0, \vec{x}_{4}\right)\right] \\
& =2 i \operatorname{Im}\left\langle\pi^{\prime}\left(\tau_{4}, \vec{y}_{4}\right) \pi\left(0, \vec{x}_{1}\right)\right\rangle s^{2}\left(\tau_{4}, \vec{y}_{4}\right) \pi\left(0, \vec{x}_{1}\right) \pi\left(0, \vec{x}_{2}\right) \pi\left(0, \vec{x}_{3}\right)+\left(x_{1} \leftrightarrow x_{2}\right)+\left(x_{1} \leftrightarrow x_{3}\right)+\left(x_{1} \leftrightarrow x_{4}\right) .
\end{aligned}
$$

From Fig. 2(a) we can see that there are two options when we calculate

$$
\mathcal{S}_{2} \equiv\left[\pi^{\prime}\left(\tau_{3}, \vec{y}_{3}\right) s^{2}\left(\tau_{3}, \vec{y}_{3}\right), \mathcal{S}_{1}\right],
$$


i.e., the position $\left(\tau_{3}, \vec{y}_{3}\right)$ can be either next to or diagonal to the position $\left(\tau_{4}, \vec{y}_{4}\right)$. Therefore, the contribution from Fig. 2(a) is

$$
\begin{aligned}
\mathcal{S}_{2}^{(1)}= & 8 i \operatorname{Im}\left\langle\pi^{\prime}\left(\tau_{4}, \vec{y}_{4}\right) \pi\left(0, \vec{x}_{1}\right)\right\rangle \times\left(\left\langle\left[\pi^{\prime}\left(\tau_{3}, \vec{y}_{3}\right) s\left(\tau_{3}, \vec{y}_{3}\right), \pi\left(0, \vec{x}_{2}\right) s\left(\tau_{4}, \vec{y}_{4}\right)\right]\right\rangle s\left(\tau_{3}, \vec{y}_{3}\right) s\left(\tau_{4}, \vec{y}_{4}\right) \pi\left(0, \vec{x}_{3}\right) \pi\left(0, \vec{x}_{4}\right)\right. \\
& \left.+\left(x_{2} \leftrightarrow x_{3}\right)+\left(x_{2} \leftrightarrow x_{4}\right)\right)+\left(x_{1} \leftrightarrow x_{2}\right)+\left(x_{1} \leftrightarrow x_{3}\right)+\left(x_{1} \leftrightarrow x_{4}\right) \\
= & -16\left[\operatorname{Im}\left\langle\pi^{\prime}\left(\tau_{4}, \vec{y}_{4}\right) \pi\left(0, \vec{x}_{1}\right)\right\rangle \operatorname{Im}\left(\left\langle\pi^{\prime}\left(\tau_{3}, \vec{y}_{3}\right) \pi\left(0, \vec{x}_{2}\right)\right\rangle\left\langle s\left(\tau_{3}, \vec{y}_{3}\right) s\left(\tau_{4}, \vec{y}_{4}\right)\right\rangle\right)\right. \\
& \left.\times s\left(\tau_{3}, \vec{y}_{3}\right) s\left(\tau_{4}, \vec{y}_{4}\right) \pi\left(0, \vec{x}_{3}\right) \pi\left(0, \vec{x}_{4}\right)+\left(x_{2} \leftrightarrow x_{3}\right)+\left(x_{2} \leftrightarrow x_{4}\right)\right]+\left(x_{1} \leftrightarrow x_{2}\right)+\left(x_{1} \leftrightarrow x_{3}\right)+\left(x_{1} \leftrightarrow x_{4}\right), \\
& \mathcal{S}_{2}^{(2)}=-4\left[\operatorname{Im}\left\langle\pi^{\prime}\left(\tau_{4}, \vec{y}_{4}\right) \pi\left(0, \vec{x}_{1}\right)\right\rangle \operatorname{Im}\left\langle\pi^{\prime}\left(\tau_{3}, \vec{y}_{3}\right) \pi\left(0, \vec{x}_{2}\right)\right\rangle s^{2}\left(\tau_{3}, \vec{y}_{3}\right) s^{2}\left(\tau_{4}, \vec{y}_{4}\right) \pi\left(0, \vec{x}_{3}\right) \pi\left(0, \vec{x}_{4}\right)\right. \\
& \left.+\left(x_{2} \leftrightarrow x_{3}\right)+\left(x_{2} \leftrightarrow x_{4}\right)\right]+\left(x_{1} \leftrightarrow x_{2}\right)+\left(x_{1} \leftrightarrow x_{3}\right)+\left(x_{1} \leftrightarrow x_{4}\right) .
\end{aligned}
$$

Now we calculate

$$
\mathcal{S}_{3} \equiv\left[\pi^{\prime}\left(\tau_{2}, \vec{y}_{2}\right) s^{2}\left(\tau_{2}, \vec{y}_{2}\right), \mathcal{S}_{2}\right] .
$$

The calculation is straightforward,

$$
\begin{aligned}
& \mathcal{S}_{3}^{(1)}=-64 i \operatorname{Im}\left\langle\pi^{\prime}\left(\tau_{4}, \vec{y}_{4}\right) \pi\left(0, \vec{x}_{1}\right)\right\rangle \operatorname{Im}\left(\left\langle\pi^{\prime}\left(\tau_{3}, \vec{y}_{3}\right) \pi\left(0, \vec{x}_{2}\right)\right\rangle\left\langle s\left(\tau_{3}, \vec{y}_{3}\right) s\left(\tau_{4}, \vec{y}_{4}\right)\right\rangle\right) \\
& \times\left[\operatorname{Im}\left(\left\langle s\left(\tau_{2}, \vec{y}_{2}\right) s\left(\tau_{3}, \vec{y}_{3}\right)\right\rangle\left\langle\pi^{\prime}\left(\tau_{2}, \vec{y}_{2}\right) \pi\left(0, \vec{x}_{3}\right)\right\rangle\right) s\left(\tau_{2}, \vec{y}_{2}\right) s\left(\tau_{4}, \vec{y}_{4}\right) \pi\left(0, \vec{x}_{4}\right)\right. \\
&\left.+\operatorname{Im}\left(\left\langle s\left(\tau_{2}, \vec{y}_{2}\right) s\left(\tau_{4}, \vec{y}_{4}\right)\right\rangle\left\langle\pi^{\prime}\left(\tau_{2}, \vec{y}_{2}\right) \pi\left(0, \vec{x}_{3}\right)\right\rangle\right) s\left(\tau_{2}, \vec{y}_{2}\right) s\left(\tau_{3}, \vec{y}_{3}\right) \pi\left(0, \vec{x}_{4}\right)\right]+\operatorname{per}\left(x_{1}, x_{2}, x_{3}, x_{4}\right), \\
& \mathcal{S}_{3}^{(2)}=-64 i \operatorname{Im}\left\langle\pi^{\prime}\left(\tau_{4}, \vec{y}_{4}\right) \pi\left(0, \vec{x}_{1}\right)\right\rangle \operatorname{Im}\left\langle\pi^{\prime}\left(\tau_{3}, \vec{y}_{3}\right) \pi\left(0, \vec{x}_{2}\right)\right\rangle \\
& \times \operatorname{Im}\left(\left\langle s\left(\tau_{2}, \vec{y}_{2}\right) s\left(\tau_{3}, \vec{y}_{3}\right)\right\rangle\left\langle s\left(\tau_{2}, \vec{y}_{2}\right) s\left(\tau_{4}, \vec{y}_{4}\right)\right\rangle\left\langle\pi^{\prime}\left(\tau_{2}, \vec{y}_{2}\right) \pi\left(0, \vec{x}_{3}\right)\right) s\left(\tau_{3}, \vec{y}_{3}\right) s\left(\tau_{4}, \vec{y}_{4}\right) \pi\left(0, \vec{x}_{4}\right)+\operatorname{per}\left(x_{1}, x_{2}, x_{3}, x_{4}\right) .\right.
\end{aligned}
$$

Therefore, we have

$$
\begin{aligned}
\mathcal{S}_{4} \equiv & {\left[\pi^{\prime}\left(\tau_{1}, \vec{y}_{1}\right) s^{2}\left(\tau_{1}, \vec{y}_{1}\right), \mathcal{S}_{3}\right] } \\
= & 256 \times\left[\operatorname{Im}\left\langle\pi^{\prime}\left(\tau_{4}, \vec{y}_{4}\right) \pi\left(0, \vec{x}_{1}\right)\right\rangle \operatorname{Im}\left(\left\langle\pi^{\prime}\left(\tau_{3}, \vec{y}_{3}\right) \pi\left(0, \vec{x}_{2}\right)\right\rangle\left\langle s\left(\tau_{3}, \vec{y}_{3}\right) s\left(\tau_{4}, \vec{y}_{4}\right)\right\rangle\right) \times \operatorname{Im}\left(\left\langle s\left(\tau_{2}, \vec{y}_{2}\right) s\left(\tau_{3}, \vec{y}_{3}\right)\right\rangle\left\langle\pi^{\prime}\left(\tau_{2}, \vec{y}_{2}\right) \pi\left(0, \vec{x}_{3}\right)\right\rangle\right)\right. \\
& \times \operatorname{Im}\left(\left\langle s\left(\tau_{1}, \vec{y}_{1}\right) s\left(\tau_{2}, \vec{y}_{2}\right)\right\rangle\left\langle s\left(\tau_{1}, \vec{y}_{1}\right) s\left(\tau_{4}, \vec{y}_{4}\right)\right\rangle\left\langle\pi^{\prime}\left(\tau_{1}, \vec{y}_{1}\right) \pi\left(0, \vec{x}_{4}\right)\right)+\operatorname{Im}\left\langle\pi^{\prime}\left(\tau_{4}, \vec{y}_{4}\right) \pi\left(0, \vec{x}_{1}\right)\right\rangle\right. \\
& \times \operatorname{Im}\left(\left\langle\pi^{\prime}\left(\tau_{3}, \vec{y}_{3}\right) \pi\left(0, \vec{x}_{2}\right)\right\rangle\left\langle s\left(\tau_{3}, \vec{y}_{3}\right) s\left(\tau_{4}, \vec{y}_{4}\right)\right\rangle\right) \times \operatorname{Im}\left(\left\langle s\left(\tau_{2}, \vec{y}_{2}\right) s\left(\tau_{4}, \vec{y}_{4}\right)\right\rangle\left\langle\pi^{\prime}\left(\tau_{2}, \vec{y}_{2}\right) \pi\left(0, \vec{x}_{3}\right)\right\rangle\right) \\
& \times \operatorname{Im}\left(\left\langle s\left(\tau_{1}, \vec{y}_{1}\right) s\left(\tau_{2}, \vec{y}_{2}\right)\right\rangle\left\langle s\left(\tau_{1}, \vec{y}_{1}\right) s\left(\tau_{3}, \vec{y}_{3}\right)\right\rangle\left\langle\pi^{\prime}\left(\tau_{1}, \vec{y}_{1}\right) \pi\left(0, \vec{x}_{4}\right)\right)+\operatorname{Im}\left\langle\pi^{\prime}\left(\tau_{4}, \vec{y}_{4}\right) \pi\left(0, \vec{x}_{1}\right)\right\rangle \operatorname{Im}\left\langle\pi^{\prime}\left(\tau_{3}, \vec{y}_{3}\right) \pi\left(0, \vec{x}_{2}\right)\right\rangle\right. \\
& \times \operatorname{Im}\left(\left\langle s\left(\tau_{2}, \vec{y}_{2}\right) s\left(\tau_{3}, \vec{y}_{3}\right)\right\rangle\left\langle s\left(\tau_{2}, \vec{y}_{2}\right) s\left(\tau_{4}, \vec{y}_{4}\right)\right\rangle\left\langle\pi^{\prime}\left(\tau_{2}, \vec{y}_{2}\right) \pi\left(0, \vec{x}_{3}\right)\right)\right. \\
& \times \operatorname{Im}\left(\left\langle s\left(\tau_{1}, \vec{y}_{1}\right) s\left(\tau_{3}, \vec{y}_{3}\right)\right\rangle\left\langle s\left(\tau_{1}, \vec{y}_{1}\right) s\left(\tau_{4}, \vec{y}_{4}\right)\right\rangle\left\langle\pi^{\prime}\left(\tau_{1}, \vec{y}_{1}\right) \pi\left(0, \vec{x}_{4}\right)\right)+\operatorname{per}\left(x_{1}, x_{2}, x_{3}, x_{4}\right) .\right.
\end{aligned}
$$


Therefore, we have

$$
\begin{aligned}
\mathcal{A}_{4}^{(a)}\left(\vec{x}_{1}, \vec{x}_{2}, \vec{x}_{3}, \vec{x}_{4}\right)= & \frac{16}{\Lambda^{8} H^{8}} \int_{-\infty}^{0} \frac{d \tau_{4}}{\tau_{4}^{3}} \cdots \int_{-\infty}^{\tau_{2}} \frac{d \tau_{1}}{\tau_{1}^{3}} \int \frac{d^{3} k_{1}}{(2 \pi)^{3}} \cdots \int \frac{d^{3} k_{4}}{(2 \pi)^{3}}(2 \pi)^{4} \delta^{4}\left(\vec{k}_{1}+\vec{k}_{2}+\vec{k}_{3}+\vec{k}_{4}\right) e^{i\left(\vec{k}_{1} \cdot \vec{x}_{1}+\cdots+\vec{k}_{4} \cdot \vec{x}_{4}\right)} \\
& \times \int \frac{d^{3} p}{(2 \pi)^{3}}\left\{\operatorname{Im}\left[\pi_{k_{1}}^{\prime}\left(\tau_{4}\right) \pi_{k_{1}}^{*}(0)\right] \operatorname{Im}\left[\pi_{k_{2}}^{\prime}\left(\tau_{3}\right) \pi_{k_{2}}^{*}\left(\tau_{3}\right) s_{\left|\vec{p}-\vec{k}_{1}\right|}\left(\tau_{3}\right) s_{\left|\vec{p}-\vec{k}_{1}\right|}^{*}\left(\tau_{4}\right)\right]\right. \\
& \times \operatorname{Im}\left[\pi_{k_{3}}^{\prime}\left(\tau_{2}\right) \pi_{k_{3}}^{*}(0) s_{\left|\vec{p}-\vec{k}_{1}-\vec{k}_{2}\right|}\left(\tau_{2}\right) s_{\left|\vec{p}-\vec{k}_{1}-\vec{k}_{2}\right|}^{*}\left(\tau_{3}\right)\right] \operatorname{Im}\left[\pi_{k_{4}}\left(\tau_{1}\right) \pi_{k_{4}}^{*}(0) s_{\left|\vec{p}+\vec{k}_{4}\right|}\left(\tau_{1}\right) s_{\left.\left|\vec{p}+\vec{k}_{4}\right|\right)}^{*}\left(\tau_{2}\right) s_{p}\left(\tau_{1}\right) s_{p}^{*}\left(\tau_{4}\right)\right] \\
& +\operatorname{Im}\left[\pi_{k_{1}}^{\prime}\left(\tau_{4}\right) \pi_{k_{1}}^{*}(0)\right] \operatorname{Im}\left[\pi_{k_{2}}^{\prime}\left(\tau_{3}\right) \pi_{k_{2}}^{*}\left(\tau_{3}\right) s_{\left|\vec{p}-\vec{k}_{2}\right|}\left(\tau_{3}\right) s_{\left|\vec{p}-\vec{k}_{2}\right|}^{*}\left(\tau_{4}\right)\right] \\
& \times \operatorname{Im}\left[\pi_{k_{3}}^{\prime}\left(\tau_{2}\right) \pi_{k_{3}}^{*}(0) s_{\left|\vec{p}-\vec{k}_{1}-\vec{k}_{2}\right|}\left(\tau_{2}\right) s_{\left|\vec{p}-\vec{k}_{1}-\vec{k}_{2}\right|}\left(\tau_{4}\right)\right] \operatorname{Im}\left[\pi_{k_{4}}\left(\tau_{1}\right) \pi_{k_{4}}^{*}(0) s_{\left|\vec{p}+\vec{k}_{4}\right|}\left(\tau_{1}\right) s_{\left.\left|\vec{p}+\vec{k}_{4}\right|\right)}^{*}\left(\tau_{2}\right) s_{p}\left(\tau_{1}\right) s_{p}^{*}\left(\tau_{3}\right)\right] \\
& +\operatorname{Im}\left[\pi_{k_{1}}^{\prime}\left(\tau_{4}\right) \pi_{k_{1}}^{*}(0)\right] \operatorname{Im}\left[\pi_{k_{3}}^{\prime}\left(\tau_{2}\right) \pi_{k_{3}}^{*}(0) s_{\left|\vec{p}+\vec{k}_{2}+\vec{k}_{4}\right|}\left(\tau_{2}\right) s_{\left|\vec{p}+\vec{k}_{2}+\vec{k}_{4}\right|}^{*}\left(\tau_{3}\right) s_{\left|\vec{p}-\vec{k}_{1}\right|}\left(\tau_{2}\right) s_{\left|\vec{p}-\vec{k}_{1}\right|}^{*}\left(\tau_{4}\right)\right] \\
& \left.\times \operatorname{Im}\left[\pi_{k_{2}}^{\prime}\left(\tau_{3}\right) \pi_{k_{1}}^{*}(0)\right] \operatorname{Im}\left[\pi_{k_{4}}^{\prime}\left(\tau_{1}\right) \pi_{k_{4}}^{*}(0) s_{\left|\vec{p}+\vec{k}_{4}\right|}\left(\tau_{1}\right) s_{\left|\vec{p}+\vec{k}_{4}\right|}^{*}\left(\tau_{3}\right) s_{p}\left(\tau_{1}\right) s_{p}^{*}\left(\tau_{4}\right)\right]\right\} \\
& +\operatorname{per}\left(x_{1}, x_{2}, x_{3}, x_{4}\right) .
\end{aligned}
$$

The trispectrum of $\zeta$ can be defined as

$$
\mathcal{A}_{4}\left(\vec{x}_{1}, \vec{x}_{2}, \vec{x}_{3}, \vec{x}_{4}\right)=\int \frac{d^{3} k_{1}}{(2 \pi)^{3}} \cdots \frac{d^{3} k_{4}}{(2 \pi)^{3}} e^{i\left(\vec{k}_{1} \cdot \vec{x}_{1}+\cdots \vec{k}_{4} \cdot \vec{x}_{4}\right)}(2 \pi)^{3} \delta^{3}\left(\vec{k}_{1}+\cdots+\vec{k}_{4}\right) \mathcal{B}_{\zeta}\left(\vec{k}_{1}, \vec{k}_{2}, \vec{k}_{3}, \vec{k}_{4}\right) .
$$

Therefore, noting that the diagram in Fig. 2(a) is dominant,

$$
\begin{aligned}
\mathcal{B}_{\zeta}\left(\vec{k}_{1}, \vec{k}_{2}, \vec{k}_{3}, \vec{k}_{4}\right)= & \frac{16}{\Lambda^{8} H^{8}} \int_{-\infty}^{0} \frac{d \tau_{4}}{\tau_{4}^{3}} \cdots \int_{-\infty}^{\tau_{2}} \frac{d \tau_{1}}{\tau_{1}^{3}} \frac{d^{3} p}{(2 \pi)^{3}} \\
& \times\left\{\operatorname{Im}\left[\pi_{k_{1}}^{\prime}\left(\tau_{4}\right) \pi_{k_{1}}^{*}(0)\right] \operatorname{Im}\left[\pi_{k_{2}}^{\prime}\left(\tau_{3}\right) \pi_{k_{2}}^{*}(0) s_{\left|\vec{p}-\vec{k}_{1}\right|}\left(\tau_{3}\right) s_{\left|\vec{p}-\vec{k}_{1}\right|}^{*}\left(\tau_{4}\right)\right]\right. \\
& \times \operatorname{Im}\left[\pi_{k_{3}}^{\prime}\left(\tau_{2}\right) \pi_{k_{3}}^{*}(0) s_{\left|\vec{p}-\vec{k}_{1}-\vec{k}_{2}\right|}\left(\tau_{2}\right) s_{\left|\vec{p}-\vec{k}_{1}-\vec{k}_{2}\right|}^{*}\left(\tau_{3}\right)\right] \operatorname{Im}\left[\pi_{k_{4}}\left(\tau_{1}\right) \pi_{k_{4}}^{*}(0) s_{\left|\vec{p}+\vec{k}_{4}\right|}\left(\tau_{1}\right) s_{\left.\left|\vec{p}+\vec{k}_{4}\right|\right)}^{*}\left(\tau_{2}\right) s_{p}\left(\tau_{1}\right) s_{p}^{*}\left(\tau_{4}\right)\right] \\
& +\operatorname{Im}\left[\pi_{k_{1}}^{\prime}\left(\tau_{4}\right) \pi_{k_{1}}^{*}(0)\right] \operatorname{Im}\left[\pi_{k_{2}}^{\prime}\left(\tau_{3}\right) \pi_{k_{2}}^{*}\left(\tau_{3}\right) s_{\left|\vec{p}-\vec{k}_{2}\right|}\left(\tau_{3}\right) s_{\left|\vec{p}-\vec{k}_{2}\right|}^{*}\left(\tau_{4}\right)\right] \\
& \times \operatorname{Im}\left[\pi_{k_{3}}^{\prime}\left(\tau_{2}\right) \pi_{k_{3}}^{*}(0) s_{\left|\vec{p}-\vec{k}_{1}-\vec{k}_{2}\right|}\left(\tau_{2}\right) s_{\left|\vec{p}-\vec{k}_{1}-\vec{k}_{2}\right|}\left(\tau_{4}\right)\right] \operatorname{Im}\left[\pi_{k_{4}}\left(\tau_{1}\right) \pi_{k_{4}}^{*}(0) s_{\left|\vec{p}+\vec{k}_{4}\right|}\left(\tau_{1}\right) s_{\left.\left|\vec{p}+\vec{k}_{4}\right|\right)}^{*}\left(\tau_{2}\right) s_{p}\left(\tau_{1}\right) s_{p}^{*}\left(\tau_{3}\right)\right] \\
& +\operatorname{Im}\left[\pi_{k_{1}}^{\prime}\left(\tau_{4}\right) \pi_{k_{1}}^{*}(0)\right] \operatorname{Im}\left[\pi_{k_{3}}^{\prime}\left(\tau_{2}\right) \pi_{k_{3}}^{*}(0) s_{\left|\vec{p}+\vec{k}_{2}+\vec{k}_{4}\right|}\left(\tau_{2}\right) s_{\left|\vec{p}+\vec{k}_{2}+\vec{k}_{4}\right|}\left(\tau_{3}\right) s_{\left|\vec{p}-\vec{k}_{1}\right|}\left(\tau_{2}\right) s_{\left|\vec{p}-\vec{k}_{1}\right|}^{*}\left(\tau_{4}\right)\right] \\
& \left.\times \operatorname{Im}\left[\pi_{k_{2}}^{\prime}\left(\tau_{3}\right) \pi_{k_{1}}^{*}(0)\right] \operatorname{Im}\left[\pi_{k_{4}}^{\prime}\left(\tau_{1}\right) \pi_{k_{4}}^{*}(0) s_{\left|\vec{p}+\vec{k}_{4}\right|}\left(\tau_{1}\right) s_{\left|\vec{p}+\vec{k}_{4}\right|}^{*}\left(\tau_{3}\right) s_{p}\left(\tau_{1}\right) s_{p}^{*}\left(\tau_{4}\right)\right]\right\} \\
& +\operatorname{per}\left(k_{1}, k_{2}, k_{3}, k_{4}\right) .
\end{aligned}
$$

Just like in the case of the three-point correlation function of $\zeta$, all of the $\tau_{i}$ integrals are mainly supported in the infrared region. Therefore, to get the leading contribution one can again Laurent expand the mode functions of $\pi$ and $s$ and collect the leading terms. Following the notation in Eq. (A12), we have to the leading order 


$$
\begin{aligned}
& \mathcal{B}_{\zeta} \simeq \frac{\pi^{4} H^{8}\left|a_{0}\right|^{8}}{256 \Lambda^{8}} \int \frac{d^{3} p}{(2 \pi)^{3}}\left\{\frac{1}{p^{3-2 \nu}\left|\vec{p}-\vec{k}_{1}\right|^{3-2 \nu}\left|\vec{p}-\vec{k}_{1}-\vec{k}_{2}\right|^{3-2 \nu}\left|\vec{p}+\vec{k}_{4}\right|^{3-2 \nu}}\right. \\
& \times \int_{-\Lambda_{4}^{(1)}}^{0} \frac{d \tau_{4}}{\left(-\tau_{4}\right)^{1-2 \nu}} \int_{-\Lambda_{3}^{(1)}}^{\tau_{4}} \frac{d \tau_{3}}{\left(-\tau_{3}\right)^{1-2 \nu}} \int_{-\Lambda_{2}^{(1)}}^{\tau_{3}} \frac{d \tau_{2}}{\left(-\tau_{2}\right)^{1-2 \nu}} \int_{-\Lambda_{1}^{(1)}}^{\tau_{2}} \frac{d \tau_{1}}{\left(-\tau_{1}\right)^{1-2 \nu}} \\
& +\frac{1}{p^{3-2 \nu}\left|\vec{p}-\vec{k}_{2}\right|^{3-2 \nu}\left|\vec{p}-\vec{k}_{1}-\vec{k}_{2}\right|^{3-2 \nu}\left|\vec{p}+\vec{k}_{4}\right|^{3-2 \nu}} \times \int_{-\Lambda_{4}^{(2)}}^{0} \frac{d \tau_{4}}{\left(-\tau_{4}\right)^{1-2 \nu}} \int_{-\Lambda_{3}^{(2)}}^{\tau_{4}} \frac{d \tau_{3}}{\left(-\tau_{3}\right)^{1-2 \nu}} \int_{-\Lambda_{2}^{(2)}}^{\tau_{3}} \frac{d \tau_{2}}{\left(-\tau_{2}\right)^{1-2 \nu}} \int_{-\Lambda_{1}^{(2)}}^{\tau_{2}} \frac{d \tau_{1}}{\left(-\tau_{1}\right)^{1-2 \nu}} \\
& \left.+\frac{1}{p^{3-2 \nu}\left|\vec{p}-\vec{k}_{1}\right|^{3-2 \nu}\left|\vec{p}+\vec{k}_{2}+\vec{k}_{4}\right|^{3-2 \nu}\left|\vec{p}+\vec{k}_{4}\right|^{3-2 \nu}} \times \int_{-\Lambda_{4}^{(3)}}^{0} \frac{d \tau_{4}}{\left(-\tau_{4}\right)^{1-2 \nu}} \int_{-\Lambda_{3}^{(3)}}^{\tau_{4}} \frac{d \tau_{3}}{\left(-\tau_{3}\right)^{1-2 \nu}} \int_{-\Lambda_{2}^{(3)}}^{\tau_{3}} \frac{d \tau_{2}}{\left(-\tau_{2}\right)^{1-2 \nu}} \int_{-\Lambda_{1}^{(3)}}^{\tau_{2}} \frac{d \tau_{1}}{\left(-\tau_{1}\right)^{1-2 \nu}}\right\} \\
& +\operatorname{per}\left(k_{1}, k_{2}, k_{3}, k_{4}\right) \text {, } \\
& =\frac{\pi^{4} H^{8}\left|a_{0}\right|^{8}}{256 \Lambda^{8}}\left(\frac{1}{2 \nu}\right)^{4} \int \frac{d^{3} p}{(2 \pi)^{3}}\left\{\frac{\mathcal{I}\left(\Lambda_{1}^{(1)}, \Lambda_{2}^{(1)}, \Lambda_{3}^{(1)}, \Lambda_{4}^{(1)}\right)}{p^{3-2 \nu}\left|\vec{p}-\vec{k}_{1}\right|^{3-2 \nu}\left|\vec{p}-\vec{k}_{1}-\vec{k}_{2}\right|^{3-2 \nu}\left|\vec{p}+\vec{k}_{4}\right|^{3-2 \nu}}\right. \\
& \left.+\frac{\mathcal{I}\left(\Lambda_{1}^{(2)}, \Lambda_{2}^{(2)}, \Lambda_{3}^{(2)}, \Lambda_{4}^{(2)}\right)}{p^{3-2 \nu}\left|\vec{p}-\vec{k}_{2}\right|^{3-2 \nu}\left|\vec{p}-\vec{k}_{1}-\vec{k}_{2}\right|^{3-2 \nu}\left|\vec{p}+\vec{k}_{4}\right|^{3-2 \nu}}+\frac{\mathcal{I}\left(\Lambda_{1}^{(3)}, \Lambda_{2}^{(3)}, \Lambda_{3}^{(3)}, \Lambda_{4}^{(3)}\right)}{p^{3-2 \nu}\left|\vec{p}-\vec{k}_{1}\right|^{3-2 \nu}\left|\vec{p}+\vec{k}_{2}+\vec{k}_{4}\right|^{3-2 \nu}\left|\vec{p}+\vec{k}_{4}\right|^{3-2 \nu}}\right\} \\
& +\operatorname{per}\left(k_{1}, k_{2}, k_{3}, k_{4}\right) \text {. }
\end{aligned}
$$

The UV cutoffs $\Lambda_{i}^{(j)}$ can be directly read off from Eq. (B12),

$$
\begin{array}{ll}
\Lambda_{1}^{(1)}=\min \left(k_{4}^{-1}, p^{-1},\left|\vec{p}+\vec{k}_{4}\right|^{-1}\right), & \Lambda_{2}^{(1)}=\min \left(k_{3}^{-1},\left|\vec{p}-\vec{k}_{1}-\vec{k}_{2}\right|^{-1},\left|\vec{p}+\vec{k}_{4}\right|^{-1}\right), \\
\Lambda_{3}^{(1)}=\min \left(k_{2}^{-1},\left|\vec{p}-\vec{k}_{1}\right|^{-1},\left|\vec{p}-\vec{k}_{1}-\vec{k}_{2}\right|\right), \quad \Lambda_{4}^{(1)}=\min \left(k_{1},\left|\vec{p}-\vec{k}_{1}\right|^{-1}, p^{-1}\right), \\
\Lambda_{1}^{(2)}=\min \left(k_{4}^{-1}, p^{-1},\left|\vec{p}+\vec{k}_{4}\right|^{-1}\right), & \Lambda_{2}^{(2)}=\min \left(k_{3}^{-1},\left|\vec{p}-\vec{k}_{1}-\vec{k}_{2}\right|^{-1},\left|\vec{p}+\vec{k}_{4}\right|^{-1}\right), \\
\Lambda_{3}^{(2)}=\min \left(k_{2}^{-1},\left|\vec{p}-\vec{k}_{2}\right|^{-1}, p^{-1}\right), & \Lambda_{4}^{(2)}=\min \left(k_{1},\left|\vec{p}-\vec{k}_{2}\right|^{-1},\left|\vec{p}-\vec{k}_{1}-\vec{k}_{2}\right|^{-1}\right), \\
\Lambda_{1}^{(3)}=\min \left(k_{4}^{-1}, p^{-1},\left|\vec{p}+\vec{k}_{4}\right|^{-1}\right), & \Lambda_{2}^{(3)}=\min \left(k_{3}^{-1},\left|\vec{p}+\vec{k}_{2}+\vec{k}_{4}\right|^{-1},\left|\vec{p}-\vec{k}_{1}\right|^{-1}\right), \\
\Lambda_{3}^{(3)}=\min \left(k_{2}^{-1},\left|\vec{p}+\vec{k}_{4}\right|^{-1},\left|\vec{p}+\vec{k}_{2}+\vec{k}_{4}\right|\right), \quad \Lambda_{4}^{(3)}=\min \left(k_{1},\left|\vec{p}-\vec{k}_{1}\right|, p^{-1}\right),
\end{array}
$$

and

$$
\begin{aligned}
\mathcal{I}\left(\Lambda_{1}, \Lambda_{2}, \Lambda_{3}, \Lambda_{4}\right)= & -\frac{1}{2} \Lambda_{123}^{4 \nu} \Lambda_{1234}^{2 \nu} \Lambda_{1}^{2 \nu}+\frac{1}{6} \Lambda_{1234}^{6 \nu} \Lambda_{1}^{2 \nu}-\frac{1}{2} \Lambda_{12}^{4 \nu} \Lambda_{123}^{2 \nu} \Lambda_{1234}^{2 \nu}+\left(\Lambda_{1} \Lambda_{12} \Lambda_{123} \Lambda_{1234}\right)^{2 \nu} \\
& +\frac{1}{6} \Lambda_{123}^{6 \nu} \Lambda_{1234}^{2 \nu}-\frac{1}{2}\left(\Lambda_{1}^{2 \nu} \Lambda_{12}^{2 \nu} \Lambda_{1234}^{4 \nu}\right)+\frac{1}{4} \Lambda_{12}^{4 \nu} \Lambda_{1234}^{4 \nu}-\frac{1}{24} \Lambda_{1234}^{8 \nu} .
\end{aligned}
$$

The definitions of $\Lambda_{12}^{(i)}, \Lambda_{123}^{(i)}$, and $\Lambda_{1234}^{(i)}$ are as in Eq. (A17). Similarly to the calculation of the trispectrum, one can capture the dominant contribution of the $p$ integral. This gives Eq. (3.4) as the leading-order result for the trispectrum with a general wave vector configuration.

\section{APPENDIX C: ONE-LOOP CORRECTION TO THE TWO-POINT FUNCTION OF $\zeta$}

The Feynman diagrams contributing to the one-loop correction to the two-point function of $\zeta$ are shown in Fig. 5. Similarly to the cases of the three- and four-point functions, the contribution from Fig. 5(a) dominates. Its contribution can be written as 


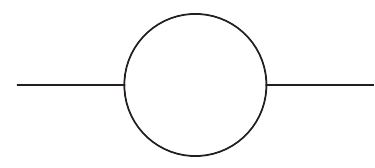

(a)

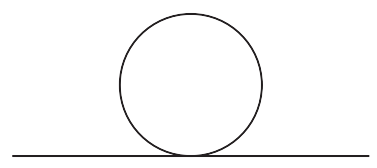

(b)

FIG. 5. One-loop diagrams for two-point functions of $\pi$.

$$
\begin{aligned}
\mathcal{A}_{2}^{(a)}\left(\vec{x}_{1}-\vec{x}_{2}\right) \\
=-\frac{1}{4 H^{4} \dot{\Lambda}^{4}} \int_{-\infty}^{0} \frac{d \tau_{2}}{\tau_{2}^{3}} \int_{-\infty}^{\tau_{2}} \frac{d \tau_{1}}{\tau_{1}^{3}} \int d^{3} y_{1} \int d^{3} y_{2} \times\left\langle\left[\pi^{\prime}\left(\tau_{1}, \vec{y}_{1}\right) s^{2}\left(\tau_{1}, \vec{y}_{1}\right),\left[\pi^{\prime}\left(\tau_{2}, \vec{y}_{2}\right) s^{2}\left(\tau_{2}, \vec{y}_{2}\right), \pi\left(0, \vec{x}_{1}\right) \pi\left(0, \vec{x}_{2}\right)\right]\right]\right\rangle \\
=-\frac{1}{4 H^{4} \dot{\Lambda}^{4}} \int_{-\infty}^{0} \frac{d \tau_{2}}{\tau_{2}^{3}} \int_{-\infty}^{\tau_{2}} \frac{d \tau_{1}}{\tau_{1}^{3}} \int d^{3} y_{1} \int d^{3} y_{2} \times\left(\left\langle\left[\pi^{\prime}\left(\tau_{1}, \vec{y}_{1}\right) s^{2}\left(\tau_{1}, \vec{y}_{1}\right), s^{2}\left(\tau_{2}, \vec{y}_{2}\right) \pi\left(0, \vec{x}_{2}\right)\right]\right\rangle\left\langle\left[\pi^{\prime}\left(\tau_{2}, \vec{y}_{2}\right), \pi\left(0, \vec{x}_{1}\right)\right]+\left(x_{1} \leftrightarrow x_{2}\right)\right)\right. \\
=-\frac{1}{4 H^{4} \dot{\Lambda}^{4}} \int_{-\infty}^{0} \frac{d \tau_{2}}{\tau_{2}^{3}} \int_{-\infty}^{\tau_{2}} \frac{d \tau_{1}}{\tau_{1}^{3}} \int d^{3} y_{1} \int d^{3} y_{2} \times(-8)\left(\operatorname{Im}\left\langle\pi^{\prime}\left(\tau_{2}, \vec{y}_{2}\right) \pi\left(0, \vec{x}_{2}\right)\right\rangle\right. \\
\left.\quad \times \operatorname{Im}\left[\left\langle\pi^{\prime}\left(\tau_{1}, \vec{y}_{1}\right) \pi\left(0, \vec{x}_{1}\right)\right\rangle\left\langle s\left(\tau_{1}, \vec{y}_{1}\right) s\left(\tau_{2}, \vec{y}_{2}\right)\right\rangle^{2}\right]+\left(x_{1} \leftrightarrow x_{2}\right)\right) \\
\approx \frac{\pi^{2} H^{4}\left|a_{0}\right|^{4}}{16 \Lambda^{4}} \int \frac{d^{3} k}{(2 \pi)^{3}} \frac{1}{k^{2}} \cos \left[\vec{k} \cdot\left(\vec{x}_{1}-\vec{x}_{2}\right)\right] \int_{-\infty}^{0} \frac{d \tau_{2}}{\tau_{2}^{3}} \int_{-\infty}^{\tau_{2}} \frac{d \tau_{1}}{\tau_{1}^{3}} \frac{d^{3} p}{(2 \pi)^{3}} \times\left(-\tau_{1}\right)^{1+2 \nu}\left(-\tau_{2}\right)^{1+2 \nu} \sin \left(k \tau_{1}\right) \sin \left(k \tau_{2}\right) \frac{1}{p^{3-2 \nu}|\vec{k}-\vec{p}|^{3-2 \nu}} \\
=\frac{H^{4}\left|a_{0}\right|^{4}}{256 \nu^{3} \Lambda^{4}} \int \frac{d^{3} k}{(2 \pi)^{3}} \frac{1}{k^{3}} e^{i \vec{k} \cdot\left(\vec{x}_{1}-\vec{x}_{2}\right)}
\end{aligned}
$$

From this one can read off the correction to the curvature perturbation,

$$
\delta \Delta_{\zeta}^{2}=\frac{H^{4}\left|a_{0}\right|^{4}}{512 \pi^{2} \nu^{3} \Lambda^{4}}
$$

[1] A. A. Starobinsky, JETP Lett. 30, 682 (1979); A. Guth, Phys. Rev. D 23, 347 (1981); A. D. Linde, Phys. Lett. 108B, 389 (1982); 114B, 431 (1982); A. Albrecht and P. Steinhardt, Phys. Rev. Lett. 48, 1220 (1982).

[2] J. M. Maldacena, J. High Energy Phys. 05 (2003) 013.

[3] T. J. Allen, B. Grinstein, and M. B. Wise, Phys. Lett. B 197, 66 (1987).

[4] N. Dalal, O. Doré, D. Huterer, and A. Shirokov, Phys. Rev. D 77, 123514 (2008).

[5] D. Baumann, S. Ferraro, D. Green, and K. M. Smith, J. Cosmol. Astropart. Phys. 05 (2013) 001.

[6] J. Gleyzes, R. de Putter, D. Green, and O. Doré, J. Cosmol. Astropart. Phys. 04 (2017) 002.

[7] H. An, M. McAneny, A. K. Ridgway, and M. B. Wise, Phys. Rev. D 97, 123528 (2018).

[8] X. Chen and Y. Wang, J. Cosmol. Astropart. Phys. 04 (2010) 027.
[9] M. H. Goroff, B. Grinstein, S. J. Rey, and M. B. Wise, Astrophys. J. 311, 6 (1986).

[10] M. McAneny, A. K. Ridgway, M. P. Solon, and M. B. Wise, Phys. Lett. B 785, 332 (2018).

[11] P. A. R. Ade et al. (Planck Collaboration), Astron. Astrophys. 571, A24 (2014).

[12] J. E. Gunn and J. R. Gott III, Astrophys. J. 176, 1 (1972).

[13] P. A. R. Ade et al. (Planck Collaboration), Astron. Astrophys. 594, A17 (2016).

[14] C. Cheung, P. Creminelli, A. L. Fitzpatrick, J. Kaplan, and L. Senatore, J. High Energy Phys. 03 (2008) 014.

[15] T. S. Bunch and P. C. W. Davies, Proc. R. Soc. A 360, 117 (1978).

[16] S. Weinberg, Phys. Rev. D 72, 043514 (2005).

[17] N. Arkani-Hamed and J. Maldacena, arXiv:1503.08043. 